\title{
CATALOGO DE LOS EJEMPLARES TIPO DE IBERODORCADION BREUNING, 1943 (COLEOPTERA, CERAMBYCIDAE) DE LA COLECCION DEL MUSEO NACIONAL DE CIENCIAS NATURALES DE MADRID
}

\author{
A. del Saz Fucho', I. Izquierdo Moya², A. Simón Sorli³, \\ J. L. Zapata de la Vega ${ }^{4}$ \& R. Gil Lara ${ }^{5}$
}

\section{RESUMEN}

A. del Saz Fucho, I. Izquierdo Moya, A. Simón Sorli, J. L. Zapata de la Vega \& R. Gil Lara. 2013. Catálogo de los ejemplares tipo de Iberodorcadion Breuning, 1943 (Coleoptera, Cerambycidae) de la colección del Museo Nacional de Ciencias Naturales de Madrid. Grael/sia, 69(1): 79-96.

Se ofrece una relación de los ejemplares tipo de Iberodorcadion conservados en la Colección de Entomología del Museo Nacional de Ciencias Naturales (MNCN) de Madrid. El presente catálogo recoge los datos de las series tipo de 125 táxones de este género, constituidas por un total de 734 ejemplares. Se designa el lectotipo de D. almarzense Escalera, 1902, y se da consideración de lectotipos a los ejemplares designados anteriormente como Holotipos de las especies D. granulipenne Escalera, 1908; D. grisescens Escalera, 1900; y D. neilense Escalera, 1902 (artículo 74.6 del Código Internacional de nomenclatura Zoológica).

Palabras clave: Coleoptera; Cerambycidae; Iberodorcadion; tipos; nomenclatura; colección; MNCN; Madrid.

\section{ABSTRACT}

A. del Saz Fucho, I. Izquierdo Moya, A. Simón Sorli, J. L. Zapata de la Vega \& R. Gil Lara. 2013. Catalog of the type specimens of Iberodorcadion Breuning, 1943 (Coleoptera, Cerambycidae) in the Entomology Collection of the Museo Nacional de Ciencias Naturales, Madrid. Grael/sia, 69(1): 79-96 (in Spanish).

The paper provides a list of the type specimens of Iberodorcadion preserved in the Entomology Collection of the Museo Nacional de Ciencias Naturales (MNCN) in Madrid. The catalog contains the data of the type series of 125 taxa of this Genus, consisting of a total of 734 specimens. The lectotype of D. almarzense Escalera, 1902 is designated, and the specimens previously recorded as holotypes of $D$. granulipenne Escalera, 1908; D. grisescens Escalera, 1900; y D. neilense Escalera, 1902, are considered as lectotypes of those three species (ICNZ, Art. 74.6).

Key Words: Coleoptera; Cerambycidae; Iberodorcadion; types; nomenclature; collections; MNCN; Madrid.

Estocolmo, 98. E-28922, Alcorcón (Madrid)-adelsaz@telefonica.net Museo Nacional de Ciencias Naturales, CSIC - izquierdo@mncn.csic.es Tarragona, 2. E-28290 Las Matas (Madrid)-simonsorli@telefonica.net Azafrán, 25. E-28760 Tres Cantos (Madrid)-jlzvega@terra.es Móstoles, 26. E-28914 Leganés (Madrid) - rafaelglara@ono.com 


\section{Introducción}

Entre los valiosos fondos científicos que conserva el Museo Nacional de Ciencias Naturales de Madrid en su Colección de Entomología (Izquierdo et al., 1997), cabe destacar la colección de Iberodorcadion Breuning, 1943 por el elevado número de especies y especímenes que contiene, estimados en más de 17.000 (Saz et al., Ref. Internet 2004), y fundamentalmente por la notable riqueza en ejemplares tipo. La Colección procede en su mayor parte de la incorporación de colecciones "históricas" reunidas por entomólogos que en los siglos XIX y comienzos del XX se ocuparon del estudio de este género (Graells, Pérez Arcas, Escalera, Bolívar, Lauffer...) a las que han venido sumándose aportaciones posteriores de otros muchos autores.

El estudio de este material tipo ha sido fundamental, y obligado, en cualquier intento de revisión del grupo, como la efectuada por E. Vives (1983). Lo ha sido asimismo en un buen número de estudios más recientes llevados a cabo por otros autores: González, 1992; González et al., 2001; Hernández, 1996; Romero, 2002; Saz, 2007a, 2007b, 2009, 2010, 2011a, 2011b; Tomé, 1998, 2001a, 2001b, 2004, 2008; Verdugo, 2001a, 2001b, 2003; Vives, 2000; Zapata, 2000.

Para este trabajo se ha llevado a cabo la revisión de la totalidad de individuos que integran la Colección del Museo, teniendo como base una exhaustiva revisión bibliográfica de las descripciones originales y de toda información complementaria publicada posteriormente a las mismas. Esta recopilación fue cotejada con la ya recogida en los archivos y bases del propio Museo, entre la que se encontraba la del material estudiado por Vives para su ya mencionada Monografía del género; y se ha completado con un seguimiento de las colecciones de los distintos autores y la identificación y estudio de las etiquetas manuscritas. Todo ello ha permitido la localización de tipos no reconocidos hasta ahora y el logro de lo que consideramos el catálogo completo de las series originales de los táxones de este género existentes en el Museo. Obviamente se han incorporando a este catálogo los tipos de táxones descritos recientemente y depositados en el centro por sus respectivos autores.

Este catálogo se enmarca en la línea de otros anteriores (Rey \& Izquierdo, 1989; Martín \& Izquierdo, 2006; Izquierdo. \& Martín, 2010) con el objetivo común de dar a conocer los tipos porta- nombres en posesión o bajo custodia del Museo Nacional de Ciencias Naturales, atendiendo con ello la Recomendación 72F del Código Internacional de Nomenclatura Zoológica sobre Responsabilidad Institucional con respecto a los ejemplares tipo.

\section{Presentación de los datos}

Una vez identificada la totalidad del material tipo, se ha confeccionado el listado de táxones, que se ofrece ordenado alfabéticamente según nombre del taxon descrito. La información referente a cada uno de ellos se ha estructurado como sigue:

Nombre del taxon. Nombre original completo tal como figura en la descripción Autor, año: páginas correspondientes a la descripción. Localidades mencionadas en la misma. $\mathrm{N}^{\circ}$ Cat., seguido del número del taxon en el Catálogo de Ejemplares Tipo de la Colección de Entomología del MNCN.

Número total de ejemplares que forman la serie. Para cada ejemplar o grupo de ejemplares: CATEGORÍA DE TIPO (número de ejemplares y sexo): información de las etiquetas, de la superior a la inferior, separándolas por un signo $+\mathrm{y}$ también [información adicional si la hubiere]. Nombre válido actual Autor, año [fuente bibliográfica de este último dato]. Y un apartado de Observaciones, en los casos en que se ha considerado pertinente.

Se ofrecen a continuación algunos comentarios que pueden facilitar la interpretación de los datos:

- En todos los casos se ha respetado la nomenclatura y grafía de la descripción original.

- Las referencias originales de los táxones reseñados se han incluido en el apartado de Bibliografía con objeto de evitar en el texto repeticiones innecesarias.

- Un único Número de Catálogo identifica a todos los ejemplares de la serie tipo de cada taxon.

- El número de ejemplares que se da para cada serie corresponde a los que se conservan en la Colección del MNCN y puede no coincidir con el total que figure en la descripción original, en los raros casos en que se dato queda reseñado.

- Se indica la categoría de tipos considerando como paratipos a los alotipos, circunstancia esta última que se menciona en los casos correspondientes.

- Figura entre corchetes toda información aclaratoria o complementaria aportada por los autores. 
- En lo referente a la disponibilidad de nombres o actos nomenclaturales, se indica en Observaciones el correspondiente artículo del Código Internacional de Nomenclatura Zoológica (CINZ).

- En cuanto a la nomenclatura actual de las diferentes especies y subespecies se han seguido fundamentalmente los criterios de Vives y Alonso-Zarazaga (2000) y González et al. (2007), con modificaciones puntuales establecidas en otros trabajos publicados o consideradas oportunas por los autores. Por otra parte, no se contemplan aquí discusiones de tipo taxonómico ni consideraciones sobre la validez de los táxones, aspecto que ha experimentado y continúa haciéndolo en la actualidad, numerosas y sucesivas modificaciones.

\section{Relación de táxones}

aberrantipes. Dorcadion Amori v. steparium a. aberrantipes Lauffer, 1911b: 44. Melés [= Uclés, corregido por el propio autor en fe de erratas]. $\mathrm{N}^{\mathrm{o}}$ Cat. 2333.

Serie tipo: 1 ej.- SINTIPO $\$$ : Dorc. var. steparius $+I b$. Baeticodorcadion iserni Perez Arcas, 1868. Zapata det. 1999. Iberodorcadion (Baeticodorcadion) isernii (Pérez Arcas, 1868). [Vives, 2000].

Obs.: Nombre no disponible al haber sido propuesto expresamente para indicar una entidad infrasubespecífica (CINZ Art. $45.5,45.6 .2$ )

abulense. Dorcadion abulense Lauffer, 1902: 1. España central, región montañosa de la provincia de Ávila, Menga. $N^{o}$ Cat. 8819.

Serie tipo: 6 ejs.- LECTOTIPO $\sigma^{x}$ : Parameras, J. Lauffer + Dorcadion abulense Lauff. + Hololectotipo + Iber. (Hispanodorc.) abulense (Lauffer) E. Vives det. 1976. 5 PARALECTOTIPOS ( $10^{\prime \prime}$ y 4 우 $ᄋ$ ): Parameras, J. Lauffer + Dorcadion abulense Lauff. + Paralectotipo + Iber. (Hispanodorc.) abulense (Lauffer), E. Vives det. 1976. Iberodorcadion (Hispanodorcadion) abulense (Lauffer, 1902). [Vives, 1983].

aguadoi. Iberodorcadion (Hispanodorcadion) aguadoi Aguado \& Tomé, 1999 (2000): 67-78. Castilla y León (España). No Cat. 8743.

Serie tipo: 2 ejs.- HOLOTIPO $\sigma^{\pi}$ : Renedo de Esgueva, Valladolid, 28.02.1998, P. Bahillo leg. + Holotypus Iberodorcadion (Hispanodorcadion) aguadoi. L. O. Aguado \& M. Tomé det. 1999. PARATIPO $\&$ : Población de Cerrato, Palencia, 07.03.1999, M. Tomé leg. + Paratypus: Iberodorcadion (Hispanodorcadion) aguadoi, M. Tomé det. 1999. Iberodorcadion (Hispanodorcadion) aguadoi Aguado \& Tomé, 2000. [Aguado y Tomé, 2000].

albarium. D. terolense sub. sp. albarium Escalera, 1902: 283. Cucalón, Teruel. No Cat. 8531.

Serie tipo: 6 ejs.- SINTIPO $\sigma^{7}: 24 / 1+$ Cucalón $+D$. terolense Esc. v. albarium Esc. $\sigma^{\pi}$ tipo + Sintipo D. terolense ssp. albarium + MNCN MADRID. SINTIPO $\sigma^{7}:$ 24/2 + Cucalón + D. terolense Esc. v. albarium Esc. $\sigma^{7}$ tipo + Sintipo D. terolense ssp. albarium. SINTIPO ㅇ: 24/3 + Teruel [en el reverso: Zapater] + D. terolense Esc. v. albarium Esc. $ᄋ$ tipo + Col. del Sr. Pérez Arcas + Iber. (Hispanodorc.) zarcoi turdetanum E. Vives det. 1977. SINTIPO \&. Cucalón, Teruel + Ib. (H.) terolense albarium Esc. E. Vives det. 1977 + sintipo. 2 SINTIPOS $\sigma^{7} \sigma^{7}$ : Col. M. Escalera. Iberodorcadion (Hispanodorcadion) terolense (Escalera, 1902) [Vives, 2000].

albolineatum. D. dejeani var. albolineatum Escalera, 1924: 200. Candeleda. N$^{\circ}$ Cat. 8793.

Serie tipo: 19 ejs.- SINTIPO $\sigma^{7}$ : Candelario $+D$. dejeani v. albolineatum Esc. $+D$. dejeani var. albolineatum Esc. Sintipo. SINTIPO $\sigma^{\pi}$ : Candelario + Iber. (Hispanodorc.) segovianum ssp. dejeani (Chevrol.) E. Vives det. $1977+$ D. dejea$n i$ var. albolineatum Esc. Sintipo. 17 SINTIPOS $\left(60^{7} \sigma^{7}\right.$ y 11 우 우): Candelario $+D$. dejeani var. albolineatum Esc. Sintipo. Iberodorcadion (Hispanodorcadion) segovianum ssp. dejeanii (Chevrolat, 1862) [Vives, 2000].

Obs. La discrepancia entre la localidad de descripción y la del etiquetado, puede corresponder a un error posiblemente en este último, dadas las numerosas capturas existentes del autor en Candeleda, donde llegó a residir.

almarzense. D. almarzense Escalera, 1902: 279-280. Almarza (Soria). No Cat. 8752.

Serie tipo: 4 ejs.- LECTOTIPO $\&: 33 / 3+$ Almarza $+D$. almarcense Esc. $\bigcirc$ tipo $+D$. almarcense Sintipo $+\mathrm{MNCN}$ Cat. Tipos No 8752. PARALECTOTIPO $\circ: 33 / 4+$ Almarza $+D$. almarcense Esc. $\sigma^{\top}$ tipo $+D$. almarcense Sintipo + MNCN Cat. Tipos $\mathrm{N}^{\circ} 8752$ [error de etiquetado relativo al sexo: el ejemplar es una hembra]. 2 PARALECTOTIPOS $\sigma^{7} \sigma^{7}$ : Almarza $+D$. almarcense Esc. $\sigma^{\top}$ tipo $+D$. almarcense Esc. Sintipo + MNCN Cat. Tipos $\mathrm{N}^{\mathrm{o}}$ 8752. Iberodorcadion (Hispanodorcadion) neilense (Escalera, 1902) [Vives, 2000].

Obs. Lectotipo aquí designado como portador único del nombre de este taxon nominal.

apicale. Dorcadion Dejeani var. montanum a. apicale Lauffer, 1911b: 54. Montañas altas de la Sierra de Béjar. No Cat. 2337.

Serie tipo: 1 ej.- SINTIPO 9 : C.J.L. $+D$. dejeani montanum a. apicale Lauff. Iberodorcadion (Hispanodorcadion) segovianum ssp. dejeanii (Chevrolat, 1862) [Vives, 2000].

Obs. Nombre no disponible (CINZ Art. 45.5, 45.6.2. Ver aberrantipes)

aragonicus. D. molitor var. arragonicus Escalera, 1924: 193. Calahorra, Logroño, Cameros. No Cat. 2332.

Serie tipo: 27 ejs.- SINTIPO $\sigma^{\pi}: 30 / 1+$ Alberite, Logroño, Rafael Vega + D. molitor v. aragonicus Esc. $\sigma^{\top}$ tipo. SINTIPO ㅇ : 30/5 + Alberite, Logroño, Rafael Vega + D. molitor v. arragonicus Esc. ㅇ tipo. SINTIPO ㅇ : 30/7 + Alberite, Logroño, Rafael Vega + D. molitor v. aragonicus Esc. ᄋ tipo + Ib. (Hisp.) molitor aragonicus (Esc.) E. Vives det. 1977. SINTIPO : 30/4 + Aragón + Col. Sr. Pérez Arcas + D. heydeni Kr. $+D$. molitor v. aragonicus Esc. O tipo. 8 SINTIPOS $\left(40^{\pi} \sigma^{\pi} \mathrm{y}\right.$ 4 우 우): Logroño, J. Ardois. SINTIPO 우: Logroño, J. Ardois + D. molitor v. arragonicus Esc. \& tipo. SINTIPO $\odot$ : Cameros, C. Bolivar. SINTIPO 우: Calahorra, J. Ardois. SINTIPO 오: Calahorra, Tutor. SINTIPO $\odot$ : Calahorra (España) + 
Dorcadion molitor. SINTIPO $\sigma^{x}$ : Calahorra + molitor v. arragonicus. 2 SINTIPOS 우 우:Calahorra (España) $+15-38+$ Regalo del Sr. Tutor + Dorcadion molitor. 4 SINTIPOS 우 우: Calahorra + 15-38 + Regalo del Sr. Tutor. 3 SINTIPOS $\left(20^{7} \sigma^{\pi}\right.$ y 1 우): Calahorra + Regalo del Sr. Tutor. Iberodorcadion (Hispanodorcadion) molitor (Fabricius, 1775).

Obs. Lapsus calami del autor. Nombre no disponible por carecer de descripción (CINZ Art. 12.1). Enumerado por Escalera, pero no descrito. Tampoco es disponible el nombre de Dorcadion (Iberodorcadion) molitor aragonicum Breuning, 1947, por haber sido propuesto por este autor como entidad infrasubespecífica (morfo) (CINZ Art.45.5, 45.6.2. Ver aberrantipes). Los 27 sintipos son atribuibles, en opinión de los autores, a la especie $I$. (H.) molitor.

arandae. Dorcadion Arandae Schramm, 1911: 306-307. Sierra de Cameros (Logroño). N ${ }^{\circ}$ Cat. 8752

Serie tipo: 5 ejs.- SINTIPO $\$$ : Sierra de Cameros, Logroño, Aranda + Iber. (Hispanodorc.) circumcinctum Chevr. E. Vives det. 1977. SINTIPO Q : Sierra de Cameros, Logroño, Aranda + Iber. (Hispanodorc.) circumcinctum 우 ssp. circumcinctum J. Romero Samper det. 3 SINTIPOS $\left(10^{7}\right.$ y 2 우 우): Sierra de Cameros, Logroño, Aranda. Iberodorcadion (Hispanodorcadion) circumcinctum (Chevrolat, 1862). [Saz, 2011a].

ardoisi. Dorcadion Ardoisi Schramm, 1909: 296-298. Sierra de Gredos (1900 m). No Cat. 8802.

Serie tipo: 4 ejs.- LECTOTIPO 9 : 58/3 + Sierra de Gredos, Dorcadion ardoisi + reg. del Sr. Ardois [en reverso 24.VII.1909] + lectotipo + Iber. castilianum ssp. ardoisi (Schr.) E. Vives det 1976. 3 PARALECTOTIPOS $\sigma^{x} \sigma^{x}$ : + Sierra de Gredos + reg. del Sr. Ardois [reverso 24.VII.1909] + paralectotipo + Iber. castilianum ssp. ardoisi (Schr.) E. Vives det 1976. [los ejemplares con etiquetas de numeración: 58/1, 58/2 y 58/4]. Iberodorcadion (Iberodorcadion) castilianum (Chevrolat, 1862) [Vives, 1983].

argenteovittatum. Dorcadion molitor v. burgense a. argenteovittatum Lauffer, 1911b: 39. Burgos, cuenca del Arlanzón y del Vega. $\mathrm{N}^{\circ}$ Cat. 2329.

Serie tipo: 1 ej.- SINTIPO $\sigma^{7}: D$. v. burgense a. argenteovittatum Lauff. Iberodorcadion (Hispanodorcadion) circumcinctum (Chevrolat, 1862) [Saz, 2011a].

Obs. Nombre no disponible (CINZ Art.45.5, 45.6.2. Ver aberrantipes).

aries. Iberodorcadion (Hispanodorcadion) aries TOMÉ \& Berger, 1999: 393-396. León. No Cat. 8655.

Serie tipo: 8 ejs.- HOLOTIPO $\sigma^{\text {T: }}$ : León, 23.III.1996, M. Tomé leg. + Holotype Iberodorcadion aries M. Tomé \& P. Berger det. 1999. 5 PARATIPOS $\left(2 \sigma^{\top} \sigma^{\top}\right.$ y 3 ㅇ ㅇ $)$ : León, 27.III.1996, M. Tomé leg. + paratype Iberodorcadion aries M. Tomé \& P. Berger det. 1999 [una $ᄋ$ etiquetada como Allotype]. 2 PARATIPOS $\sigma^{7} \sigma^{7}$ : León 30.III.1997, M. Tomé leg. + paratype Iberodorcadion aries M. Tomé \& P. Berger det. 1999. Iberodorcadion (Hispanodorcadion) aries Tomé y Berger, 1999 [Tomé y Berger, 1999].

aureotomentosum. Dorcadion Uhagoni a. aureotomentosum Lauffer, 1911a: 30. Cuenca. $\mathrm{N}^{\circ}$ Cat. 8816.

Serie tipo: 1 ej.- SINTIPO 을 $D$. uhagoni a. aureotomentosum Lauff. + Cuenca, Lauffer + Iber. (Hispanodorc.) uhagoni
(P.A.) E. Vives det. $1977+$ D. uhagoni v. aureotomentosum Lauff. Holotipo. Iberodorcadion (Hispano-dorcadion) uhagonii (Pérez Arcas, 1868) [Saz, 2007b].

Obs. Nombre no disponible (CINZ Art.45.5, 45.6.2. Ver aberrantipes).

auripenne. D. auripenne Escalera, 1908: 334-335. Cuenca de Ayllón. $\mathrm{N}^{\mathrm{o}}$ Cat. 8749.

Serie tipo: 5 ejs.- 5 SINTIPOS $\left(30^{\pi} \sigma^{\pi}, 2\right.$ 우 우): R. Ayllón $+D$. auripenne Esc. $\sigma^{\prime \prime}+$ Sintipo D. auripenne Esc. [todos los ejemplares con etiquetas de numeración: $51 / 1$ a 51/4; y 51/6]. Iberodorcadion (Hispanodorcadion) graellsii ssp. cinereum (Escalera, 1901) [Vives, 2000].

becerrae. Dorcadion Becerrae Lauffer, 1901: 90-91. Soria. No Cat. 8815 .

Serie tipo: 14 ejs.- LECTOTIPO $\sigma^{7}$ : Soria, Becerra + ejemplar descripción $+D$. becerrae Lauff. + lectotipo + Hispanodorcadion seguntianum ssp. becerrae (Lauff.) E. Vives det. 1977 + MNCN MADRID + Iberodorcadion becerrae Lauffer M. Tomé det. 1998. 2 PARALECTOTIPOS ( $\sigma^{7}$ y O ): Soria + Typ. descrip. + paralectotipo + Iber. (Hispanodorc.) seguntianum ssp. becerrae (Lauff.) E. Vives det. 1977 + MNCN MADRID + Iberod . becerrae Lauffer M. Tomé det. 1998. PARALECTOTIPO: Soria + Typ. descrip. + paralectotipo + Iber. (Hispanodorc.) seguntianum ssp. becerrae (Lauff.) E. Vives det. 1977 + MNCN MADRID + Iberodorcadion becerrae Lauffer M. Tomé det. 1998. 4 PARALECTOTIPOS ( $2 \sigma^{7} \sigma^{7}$ y 2 ㅇ $q$ ): Soria + Typ. descrip. + paralectotipo + Iber. (Hispanodorc.) seguntianum ssp. becerrae (Lauff.) E. Vives det. 1977 + MNCN MADRID + Iberod. becerrae Lauffer M. Tomé det. 1998. 6 PARALECTOTIPOS $\left(30^{\pi} \sigma^{\pi}\right.$ y 3 우 우): Soria + ejemplar de descripción + paralectotipo + Hispan. seguntianum ssp. becerrae (Lauff.) E. Vives det. 1977 + MNCN MADRID + Iberod. becerrae Lauffer, M. Tomé det. 1998. Iberodorcadion (Hispanodorcadion) becerrae (Lauffer, 1901) [González et al., 2007].

bolivari. Dorcadion Bolivari Lauffer, 1898: 107. Quero (Ciudad Real) [error en etiqueta, la provincia correcta es Toledo]. N ${ }^{\circ}$ Cat. 8784.

Serie tipo: 3 ejs.- LECTOTIPO $\sigma^{7}: 47 / 1+$ Quero, Bolívar $+D$. bolivari $\sigma^{7}$ Lauff. $+D$. bolivari $\sigma^{7}$ Esc. vidit + lectotipo + Iber. (Hispanodorc.) bolivari (Lauffer) E. Vives det. 1976. PARALECTOTIPO $0^{7}:$ : 47/5 + Quero, Bolívar + D. bolivari $\sigma^{7}$, Esc. vidit + paralectotipo + Iber. (Hispanodorc.) bolivari (Lauffer) E. Vives det. 1976. PARALECTOTIPO + : 47/13+ Quero, Bolívar $+D$. bolivari ㅇ Lauff. $+D$. bolivari 오 Esc. vidit + Iber. (Hispanodorc.) bolivari (Lauffer) E. Vives det. Iberodorcadion (Hispanodorcadion) bolivari (Lauffer, 1898) [Vives, 1983].

bouvieri. Dorcadion bouvieri Escalera, 1900: 232-233. Galera (Granada). N ${ }^{\circ}$ Cat. 8783.

Serie tipo: 8 ejs.- SINTIPO $\sigma^{\pi}: 46 / 2+$ Galera, Granada, Esc., $1900+$ D. bouvieri Esc. $\sigma^{7}$ Tipo $+I b$. (Hispanod.) fuentei (Pic), Zapata det. $1998+$ D. bouvieri Esc. Sintipo. 2 SINTIPOS 우 옹 Galera, Granada, Esc., $1900+$ D. bouvieri Esc. o Tipo $+D$. bouvieri Esc. Sintipo [ambos ejemplares con etiqueta de numeración: 46/3 y 46/4]. SINTIPO 우: Galera, Granada, Esc., $1900+$ D. bouvieri Esc. + D. bouvieri Esc. Sintipo + Ib. (Hispanod.) fuentei Pic, J. L. Zapata det. 1998. 
SINTIPO $\sigma^{x}$ : Bouvieri Escalera Typo [en reverso: Galera, Granada, 5.900]. 2 SINTIPOS $\sigma^{7} \sigma^{7}$ : Galera, Granada, Esc., $1900+$ D. bouvieri Esc. + D. bouvieri Esc. Sintipo. SINTIPO ㅇ : Bouvieri Typo + D. bouvieri Esc. + Ib. (Hispanod.) fuentei Zapata det. 1998. Iberodorcadion (Hispanodorcadion) fuentei (Pic, 1899) [Zapata, 2000].

brunneofasciatum. Dorcadion hispanicum var. brunneofasciatum Lauffer, 1901: 94. Sierra de Guadarrama. No Cat. 2327.

Serie tipo: 1 ej.- SINTIPO $\odot$ : Peñalara $+D$. hispanicum v. bruneofasciatum Lauff. Iberodorcadion (Hispanodorcadion) perezi ssp. hispanicum (Mulsant, 1851) [Hernández, 2000].

burgense. Dorcadion molitor v. burgense Lauffer, 1911a: 3536. Alrededores de Burgos, cuenca de los ríos Arlanzón y Vega. $\mathrm{N}^{\mathrm{o}}$ Cat. 2328.

Serie tipo: 11 ejs.- SINTIPO $\sigma^{\pi}$. D. molitor v. burgense Lauff. SINTIPO : D. molitor burgense + Prov. de Burgos, Lauffer. 9 SINTIPOS ( $80^{x} \sigma^{7}$ y 1 ㅇ $)$ : Prov. de Burgos, Lauffer. Iberodorcadion (Hispanodorcadion) circumcinctum (Chevrolat, 1862) [Vives, 1983].

caesaraugustae. Dorcadion molitor v. Caesaraugustae Lauffer, 1911a: 34. Alrededores de Zaragoza. No Cat. 7706.

Serie tipo: 1 ej.- SINTIPO $\sigma^{x}:$ D. molitor caesaraugustae Lauf. + Zaragoza, P. Navas + Ib. (Hispanodorc.) molitor navasi Esc. E. Vives det. 1977 + Dorcadion molitor caesaraugustae Lauff. Sintipo. Iberodorcadion (Hispanodorcadion) molitor ssp. navasi (Escalera, 1900) [Vives, 1983].

carbonicum. D. dejeani var. carbonicum Escalera, 1924: 200. Candeleda. N ${ }^{\circ}$ Cat. 8792.

Serie tipo: 4 ejs.- SINTIPO $ᄋ$ : Candelario + dejeani v. carbonicum Esc. + D. dejeani var. carbonicum Esc. Sintipo. SINTIPO 울 Candelario + Iber. (Hispanodorc.) segovianum ssp. dejeani (Chevrol.) E. Vives det. $1977+$ D. dejeani var. carbonicum Esc. Sintipo. SINTIPO + : Candelario $+D$. dejeani var. carbonicum Esc. Sintipo. SINTIPO $ᄋ:$ D. dejeani var. carbonicum Esc. Sintipo. Iberodorcadion (Hispanodorcadion) segovianum ssp. dejeanii (Chevrolat, 1862) [Vives, 2000].

Obs. La discrepancia entre la localidad de descripción y la del etiquetado puede corresponder a un error, posiblemente en este último, dadas las numerosas capturas existentes del autor en Candeleda, donde llegó a residir.

cazurroi. Dorcadion Graellsi var. Cazurroi Lauffer, 1901: 9192. Cercedilla región montana inferior. $\mathrm{N}^{\mathrm{o}}$ Cat. 8814.

Serie tipo: 12 ejs.- SINTIPO $\sigma^{\prime}$. Cercedilla, J. Lauffer + Graellsi Graells v. cazurroi Lauff. + D. graellsi var. cazurroi Lauff. Sintipo. 10 SINTIPOS $\left(5 \sigma^{7} \sigma^{7}, 5 \%\right.$ Q $)$ : Cercedilla, J. Lauffer + D. graellsi var. cazurroi Lauff. Sintipo. SINTIPO $ᄋ$ : Cercedilla, J. Lauffer + graellsi a. cazurroi Lauff. Iberodorcadion (Hispanodorcadion) graellsii ssp. longipenne (Chevrolat, 1862) [Vives, 2000].

cebollerense. Dorcadion hispanicum v. cebollerense Lauffer, 1911b: 40-41. Somosierra, La Cebollera (provincia de Madrid). $\mathrm{N}^{\circ}$ Cat. 8804.

Serie tipo: 34 ejs.- SINTIPO $\$$ : Somosierra, Lauffer $+D$. hispanicum v. cebollerense Lauff. Sintipo. SINTIPO $\sigma^{7}$ : La Cebollera $+D$. hispanicum cebollerense Lauff. $+D$. hispanicum var. cebollerense Lauff. Sintipo. SINTIPO $\sigma^{7}$. Somosierra, Lauffer $+D$. hispanicum v. cebollerense Lauffer $+D$. hispanicum v. cebollerense Lauff. Sintipo. 3 SINTIPOS $\sigma^{x} \sigma^{x}: D$. hispanicum cebollerense $+D$. hispanicum v. cebollerense Lauff. Tipo. 28 SINTIPOS (210 $\sigma^{7}$ y 7 우 우): Somosierra, Lauffer. Iberodorcadion (Hispanodorcadion) perezi ssp. hispanicum (Mulsant, 1851) [Hernández, 2000].

cinereum. D. Graellsi Sub-especie cinereum Escalera, 1901a: 81. Robregordo 1500 m. [= Dorcadion Graellsi var. cinereum Lauffer, 1901: 92]. No Cat. 8748.

Serie tipo: 2 ejs.- LECTOTIPO $\sigma^{\top}$ : Somosierra, J. Lauffer + lectoholotipo + Dorcadion graellsi v. cinereum Lauff. + Iber. (Hispanodor.) graellsi ssp. cinereum (Lauffer) E. Vives det. 1977. PARALECTOTIPO O : Somosierra, J. Lauffer + paralectotipo + Iber. (Hispanodor.) graellsi v. cinereum Lauff. E. Vives det 1977 + MNCN MADRID. Iberodorcadion (Hispanodorcadion) graellsii ssp. cinereum (Escalera, 1901) [Vives, 2000].

Obs. Aunque Escalera menciona este taxon como de Lauffer, cuyo artículo aparece en la misma publicación y a continuación del suyo (p.92), es Escalera el autor del taxon por atribuirle una categoría superior a la de Lauffer (CINZ Art. 24.1).

circumalbum. D. villosladense var. circumalbum Escalera, 1924: 199-200. Cameros, Logroño. $\mathrm{N}^{\circ}$ Cat. 8787.

Serie tipo: 4 ejs.- SINTIPO $\sigma^{x}:$ Logroño $+D$. circumalbum Esc. + D. villosladense v. circumalbum Esc. Sintipo + MNCN Cat. Tipos $N^{\circ}$ 8787. SINTIPO $\sigma^{7}$ : Cameros, Logroño $+D$. villosladense v. circumalbum Esc. Sintipo + MNCN Cat. Tipos $\mathrm{N}^{\circ}$ 8787. SINTIPO $ᄋ$ : Cameros, C. Bolivar $+I b$. (Hisp.) neilense v. subbrevipenne (Pic) E. Vives det. $1977+$ D. villosladense v. circumalbum Esc. Sintipo + MNCN Cat. Tipos No 8787. SINTIPO $\&$ : Cameros, C. Bolívar + D. villosladense v. circumalbum Esc. Sintipo + MNCN Cat. Tipos $\mathrm{N}^{\circ} 8787$. Iberodorcadion (Hispanodorcadion) neilense (Escalera, 1902) [Vives, 2000].

coelloi. Iberodorcadion (Baeticodorcadion) coelloi Verdugo, 1995: 9-21. Conil y Chiclana de la Frontera (Cádiz). No Cat. 11968.

Serie tipo: 2 ejs.- PARATIPOS ( $\sigma^{\pi}$, $\left.\bigcirc\right)$ : Conil, Cádiz, Hispania, 18.feb.1994, Pedro Coello leg. + Paratypus Iberodorcadion coelloi n. sp. A. Verdugo det. 1994. Iberodorcadion (Baeticodorcadion) coelloi (Verdugo, 1996) [Verdugo, 1996].

consanguineum. Dorcadion consanguineum Lauffer, 1911b: 40. Montes Carpetanos en el límite de la provincia de Madrid y la de Segovia. N $^{\circ}$ Cat. 2326.

Serie tipo: 1 ej.- SINTIPO $\sigma^{\top}$ : Sierra de Guadarrama, J. Lauffer + Dorcadion consanguineum Lauff. Iberodorcadion (Hispanodorcadion) graellsii (Graells, 1858) [Vives, 2000].

costatum. D. Almarzense Var. costatum Escalera, 1902: 280. Almarza. $N^{\circ}$ Cat. 8777.

Serie tipo: 2 ejs.- SINTIPOS ( $\sigma^{\pi}$,, ) $)$ Almarza $+D$. almarcense v. costatum Esc. tipo $+D$. almarcense v. costatum Esc. Sintipo + MNCN Cat. Tipos No 8777. [Los ejemplares con etiquetas de numeración: 33/5 y 33/6]. Iberodorcadion (Hispanodorcadion) neilense (Escalera, 1902) [Vives, 2000]. 
decemvittatum. Dorcadion Martinezi a. 10-vittatum Lauffer, 1911a: 32. Madrid. N$^{\circ}$ Cat. 8811.

Serie tipo: 1 ej.- SINTIPO + : Madrid + Dorcadion martinezi a. 10-vittatum Lauff. + D. martinezi ab. decemvittatum Lauff. Sintipo. Iberodorcadion (Hispanodor-cadion) martinezii (Pérez Arcas, 1874). [Saz, 2007b].

Obs. Nombre no disponible (CINZ Art.45.5, 45.6.2. Ver aberrantipes).

decemvittatum. Dorcadion molitor v. burgense a. decemvittatum Lauffer, 1911b: 39-40. Burgos, cuencas del Arlanzón y del Vega. $\mathrm{N}^{\circ}$ Cat. 2340.

$\mathrm{N}^{\mathrm{o}}$ de Tipos: 1 ej.- SINTIPO 우:D. v. burgense a. decemlineatum Lauff. Iberodorcadion (Hispanodorcadion) circumcinctum (Chevrolat, 1862) [Saz, 2011a].

Obs. Nombre no disponible (CINZ Art.45.5, 45.6.2. Ver aberrantipes).

demandense. D. Demandense Escalera, 1902: 286-287. Pineda (Sierra de la Demanda). N ${ }^{\circ}$ Cat. 8824.

Serie tipo: 5 ejs.- LECTOTIPO $\sigma^{x}: 21 / 1+\mathrm{S}^{\mathrm{a}}$ Demanda + D. demandense Esc. $\sigma^{\pi}$ typo + lectotipo + Iber. (Hispanodorc.) demandense (Esc.) E. Vives det. 1977. PARALECTOTIPO $\sigma^{7}: 21 / 2+S^{a}$ Demanda $+D$. demandense Esc. $\sigma^{7}$ typo + paralectotipo + Iber. (Hispanodorc.) demandense (Esc.) E. Vives det. 1977. 3 PARALECTOTIPOS $ᄋ$ 오 : $\mathrm{S}^{\mathrm{a}}$ Demanda $+D$. demandense Esc. $ᄋ$ typo + paralectotipo + Iber. (Hispanodorc.) demandense (Esc.) E. Vives det. 1977. [Los ejemplares con etiquetas de numeración: 21/4 a 21/6]. Iberodorcadion (Hispanodorcadion) albicans ssp. demandense (Escalera, 1902) [Vives, 1983].

denudatum. D. ignotum var. denudatum Escalera, 1911: 81. Teruel. $N^{\circ}$ Cat. 8745.

Serie tipo: 15 ejs.- 2 SINTIPOS ( $\sigma^{\pi}$, 우 ): Teruel, A. Sanz + Dorcadion v. denudatum Esc. 6 SINTIPOS ( $30^{\top} \sigma^{\prime}, 3$ ㅇ 우): Teruel, A. Sanz. 7 SINTIPOS ( $3 \sigma^{7} \sigma^{7}, 4$ 우 우): Teruel, A. Sanz + MNCN MADRID. Iberodorcadion (Hispanodorcadion) zarcoi ssp. turdetanum (Lauffer, 1911) [Vives, 2000].

dispersepunctatum. D. dispersepunctatus Escalera, 1924: 195196. Montalbanejo, Osa de la Vega. $N^{\circ}$ Cat. 8794.

Serie tipo: 2 ejs.- SINTIPO $\sigma^{7}:$ Montalbanejo $+D$. dispersepunctatum Escalera, Sintipo. SINTIPO $\sigma^{7}$ : Osa de la Vega + Iber. (Baeticodorcadion) iserni (Per. A.) E. Vives det. $1976+D$. dispersepuntatum Escalera, Sintipo. Iberodorcadion (Baeticodorcadion) isernii (Pérez Arcas, 1868). [Vives, 1983].

Obs. Lapsus calami del autor.

distinctum. Dorcadion Perezi a. distinctum Lauffer, 1911c: 74. Alrededores del Escorial. No Cat. 2325.

Serie tipo: 2 ejs.- SINTIPO $\sigma^{7}$. El Escorial, J. Lauffer $+D$. perezi a. distinctum Lauffer. SINTIPO $\sigma^{x}: D$. perezi a. distinctum Lauff. Iberodorcadion (Hispanodorcadion) perezi (Graells, 1849) [Vives, 2000].

Obs. Nombre no disponible (CINZ Art.45.5, 45.6.2. Ver aberrantipes).

dorsigerum. Dorcadion Martinezi a. dorsigerum Lauffer, 1911a: 31-32. Madrid. No Cat. 8810

Serie tipo: 4 ejs.- SINTIPO $\sigma^{\pi}$ : Madrid, J. Lauffer + Dorcadion martinezi a. dorsigerum Lauff. $+D$. martinezi ab. dorsigerum Lauff. Sintipo. 3 SINTIPOS (10 y 2 우 우): Madrid J. Lauffer $+D$. martinezi ab. dorsigerum Lauff. Sintipo. Iberodorcadion (Hispanodorcadion) martinezii (Pérez Arcas, 1874) [Saz, 2007b].

Obs. Nombre no disponible (CINZ Art.45.5, 45.6.2. Ver aberrantipes).

ebeninum. Dorcadion Ghilianii 우 var. ebeninum Lauffer, 1901: 96-97. [En la descripción original no se indica localidad]. $\mathrm{N}^{\circ}$ Cat. 8818.

Serie tipo: 1 ej.- SINTIPO $\$$ : D. Reichei v. ebeninum Lauffer + Sierra de Guadarrama, J. Lauffer + D. ghiliani var. ebeninum Lauff. Sintipo. Iberodorcadion (Hispanodorcadion) perezi ssp. ghilianii (Chevrolat, 1862) [González et al., 2007].

escalerae. Dorcadion Escalerae Lauffer, 1901: 89. Monte Cauno. $\mathrm{N}^{\mathrm{o}}$ Cat. 10072.

Serie tipo: 1 ej.- HOLOTIPO $\sigma^{\mathrm{x}}$ : Moncayo, VII.900 + D. escalerae Lauff. + holotipo + Iber. (Baeticodorcadion) spinolae caunense (Lauff.) E. Vives det. 1976. Iberodorcadion (Iberodorcadion) spinolae ssp. caunense (Lauffer, 1910). [Vives, 1983].

Obs. El propio autor (1910: 91-92) es quien propone el cambio de nombre a spinolae v. caunense.

ferdinandi. Dorcadion ferdinandi Escalera, 1900: 235. Huéscar, Galera (Granada). No Cat. 8782.

Serie tipo: 4 ejs.- LECTOTIPO $\sigma^{x}: 6 / 4+$ Galera, Granada, Esc. $1900+D$. ferdinandi Esc. $\sigma^{7}$ tipo + lectotipo $+I b$. (Hispanodorcadion) ferdinandi E. Vives det. 1976. 3 PARALECTOTIPOS (2 $\sigma^{7} \sigma^{7}, 1$ 우 ): Galera, Granada, Esc. $1900+D$. ferdinandi Esc. tipo + paralectotipo $+I b$. (Hispanodorcadion) ferdinandi E. Vives det. 1976. [Los paralectotipos con etiquetas de numeración: 6/1 a 6/3]. Iberodorcadion (Hispanodorcadion) ferdinandi (Escalera, 1900). [Verdugo, 1995].

ferruginipes. Dorcadion molitor v. burgense a. ferruginipes Lauffer, 1911a: 36. Alrededores de Burgos y cuencas de los ríos Arlanzón y Vega. $\mathrm{N}^{\mathrm{o}}$ Cat. 8753.

Serie tipo: 1 ej.- SINTIPO $\sigma^{x}: D$. molitor v. burgense a. ferruginipes Lauf. + Arlanzón + Dorcadion molitor var. ferruginipes Lauff. Sintipo. Iberodorcadion (Hispanodorcadion) circumcinctum (Chevrolat, 1862). [Saz, 2011a].

Obs. Nombre no disponible (CINZ Art.45.5, 45.6.2. Ver aberrantipes).

fuentei. Dorcadion fuentei Pic, 1899: 81. Pozuelo (Ciudad Real). No Cat. 2324.

Serie tipo: 1 ej.- SINTIPO $\sigma^{\pi} .40 / 1+$ Pozuelo de Cva. $+D$. fuentei $\sigma^{7}$ cotipo Pic, Esc. Vidit. Iberodorcadion (Hispanodorcadion) fuentei (Pic, 1899) [Vives, 1983].

fulvipes. Dorcadion Dejeani var. submontanum a. fulvipes Lauffer, 1911b: 55. Candelario y alrededores. No Cat. 2338.

Serie tipo: 1 ej.- SINTIPO $0^{\text {: }}$ : C.J.L. + D. dejeani submontanum a. fulvipes Lauff. Iberodorcadion (Hispanodorcadion) segovianum ssp. dejeanii (Chevrolat, 1862) [Vives, 2000].

Obs. Nombre no disponible (CINZ Art.45.5, 45.6.2. Ver aberrantipes). 
funerarium. Dorcadion Dejeani var. submontanum a. funerarium Lauffer, 1911b: 55. Candelario. No Cat. 2323.

Serie tipo: 2 ejs.- 2 SINTIPOS 우 우: D. dejeani submontanum a. funerarium Lauff. Iberodorcadion (Hispanodorcadion) segovianum ssp. dejeanii (Chevrolat, 1862) [Vives, 2000].

Obs. Nombre no disponible (CINZ Art.45.5, 45.6.2. Ver aberrantipes).

gagatum. Dorcadion molitor v. burgense a. gagatum Lauffer, 1911b: 39. Burgos, cuencas del Arlanzón y Vega. N ${ }^{\circ}$ Cat. 2330.

Serie tipo: 1 ej.- SINTIPO $ᄋ:$ D. v. burgense a. gagatum Lauff. Iberodorcadion (Hispanodorcadion) circumcinctum (Chevrolat, 1862) [Saz, 2011a].

Obs. Nombre no disponible (CINZ Art.45.5, 45.6.2. Ver aberrantipes).

giganteum. D. Lorquini var. giganteum Escalera, 1911: 82. S Nevada frente a Fiñana, Pto. de la Ragua. N ${ }^{\circ}$ Cat. 8797.

Serie tipo: 6 ejs.- 2 SINTIPOS $\left(\sigma^{\pi} \sigma^{\pi}\right)$ : Sierra Nevada VII.1903. Escalera $+D$. lorquini v. giganteus Esc. $\sigma^{\pi}$ tipo $+D$. lorquini var. giganteum Esc. [etiquetas de numeración 17/1 y 17/2]. 2 SINTIPOS ( $\circ$ ㅇ ): Sierra Nevada VII.1903. Escalera $+D$. lorquini! v. giganteus Esc. ㅇ tipo $+D$. lorquini. [etiquetas de numeración 17/3 y 17/4] 2 SINTIPOS $\sigma^{x} \sigma^{x}$ : Pto. de la Ragua $+D$. lorquini var. giganteum Esc. Sintipo. Iberodorcadion (Baeticodorcadion) lorquinii (Fairmaire, 1855) [Vives, 2000].

graellsii. Dorcadion graellsii Graells, 1858: 91. El Escorial y La Granja. N ${ }^{\circ}$ Cat. 8747.

Serie tipo: 1 ej.- NEOTIPO $\sigma^{\top}$. La Granja VII.1907, Escribano + neoholotipo $+I b$. (Hispanodorcadion) graellsi Graells, Vives det. 1977 + MNCN MADRID. Iberodorcadion (Hispanodorcadion) graellsii (Graells, 1858) [Vives, 1983].

Obs. La hembra designada como Neoalotipo por Vives (1983) no tiene condición de portanombre por lo que no forma parte de la serie tipo (CINZ Recom. 72A).

granulipenne. D. granulipenne Escalera, 1908: 337. Cuenca del Zapardiel. No Cat. 8764.

Serie tipo: 2 ejs.- LECTOTIPO ㅇ: Fte. el Saz + D. granulipenne Esc. + hololectotipo + Ib. (Hispanod.) abulense ssp. granulipenne E. Vives det. 1977. PARALECTOTIPO $\sigma^{7}$ : Fte. el Saz. Iberodorcadion (Hispanodorcadion) abulense ssp. granulipenne (Escalera, 1908) [Vives, 1983].

Obs. La localidad de captura debe ser Fuente el Sauz (Ávila), erróneamente consignada en el etiquetado. Dado que la descripción original del taxon no permite inferir la existencia de un único ejemplar, la mención de Holotipo (Vives, 1983) equivale a una designación de Lectotipo (CINZ Recom. 73F, Art. 74.6).

gredosanum. Dorcadion segovianum var. gredosanum Lauffer, 1911b: 50-51. Sierra de Gredos, Villarejo del Valle, Navarredonda, Navalperal del Tormes, Sierra Serrota, Sierra de Guadalupe. Gilgarcía. N ${ }^{\circ}$ Cat. 8809.

Serie tipo: 1 ej.- SINTIPO $\sigma^{\pi}$ : Navarredonda $+D$. dejeani var. gredense Lauff. $+D$. segovianum v. gredosanum Lauff. Sintipo. Iberodorcadion (Hispanodorcadion) segovianum (Chevrolat, 1862) [Vives, 2000]. griseicolle. Dorcadion molitor v. burgense a. griseicolle Lauffer, 1911b: 39. [En la descripción original no se indica localidad]. No Cat. 2331.

Serie tipo: 1 ej.- SINTIPO $\sigma^{x}: D$. v. burgense a. griseicolle Lauff. Iberodorcadion (Hispanodorcadion) circumcinctum (Chevrolat, 1862) [Saz, 2011a].

Obs. Nombre no disponible (CINZ Art.45.5, 45.6.2. Ver aberrantipes).

griseopubescens. Dorcadion hispanicum v. griseo-pubescens Lauffer, 1911b: 41-42. Sierra de Guadarrama. No Cat. 8803.

Serie tipo: 1 ej.- SINTIPO $0^{x}$ : Sierra de Guadarrama $+D$. hispanicum v. griseopubescens Lauff. + Iber. (Hispanodorc.) graellsi (Graells) E. Vives det. $1977+$ D. hispanicum var. griseopubescens Lauff. Sintipo. Iberodorcadion (Hispanodorcadion) perezi ssp. hispanicum (Mulsant, 1851) [Hernández, 2000].

grisescens. Dorcadion grisescens Escalera, 1900: 240-241. Castril (Granada). N ${ }^{\circ}$ Cat. 8798.

Serie tipo: 1 ej.- LECTOTIPO $\sigma^{\top}$ : Castril + Dorcadion grisescens Esc. $1900+$ lectotipo + lectotipo [en reverso: citado como holotipo por Vives 1983] + I. (Baeticodorcadion) escalerai (Pic) E. Vives det $1977+$ MNCN MADRID + Iberodorcadion mus (Rosenhauer 1856) A. Verdugo det 1997. Iberodorcadion (Baeticodorcadion) mus (Rosenhauer, 1856) [Verdugo, 2001a].

Obs. Dado que la descripción original del taxon no permite inferir la existencia de un único ejemplar, la mención de Holotipo (Vives, 1983) equivale a una designación de Lectotipo (CINZ Recom. 73F, Art. 74.6).

griseum. D. umbripenne var. griseum Escalera, 1911: 83. [En la descripción original no se indica localidad]. $\mathrm{N}^{\circ}$ Cat. 8770.

Serie tipo: 3 ejs.- 2 SINTIPOS $\sigma^{\prime \prime}$, $ᄋ$ : Villatoro, J. Ardois + D. umbripenne var. griseum Esc. Sintipo. SINTIPO 우 Menga, J. Ardois + D. umbripenne var. griseum Esc. Sintipo. Iberodorcadion (Hispanodorcadion) abulense (Lauffer, 1902) [Vives, 2000].

grustani. Iberodorcadion (Hispanodorcadion) grustani González, 1992: 3-8. Sierra de Cucalón (Teruel). Nº Cat. 7729.

Serie tipo: 2 ejs.- PARATIPOS $\sigma^{\pi}$, $\$$ : Barrachina, Teruel, C. González leg. Paratipo. Iberodorcadion (Hispanodorcadion) grustani C. González det. 1992. Iberodorcadion (Hispanodorcadion) grustani González, 1992. [González, 1992].

hispanoloide. D. umbripenne var. hispanoloide Escalera, 1911: 84. [En la descripción original no se indica localidad]. $\mathrm{N}^{\mathrm{o}}$ Cat. 8774 .

Serie tipo: 8 ejs.- SINTIPO $\sigma^{7}$ : Pto. de Chia + D. umbripenne var. hispanoloide Esc. Sintipo. 7 SINTIPOS $\left(60^{7} \sigma^{7} \mathrm{y}\right.$ 1 ㅇ ): Villatoro, J. Ardois + D. umbripenne var. hispanoloide Esc. Sintipo. Iberodorcadion (Hispanodorcadion) abulense (Lauffer, 1902) [Vives, 2000].

hortigüelae. Dorcadion Becerrae a. Hortigüelae Lauffer, 1911a: 31. Covarrubias, sudeste de la provincia de Burgos. N ${ }^{\circ}$ Cat. 2346.

Serie tipo: 1 ej.- SINTIPO $\sigma^{x}: D$. becerrae a. hortiguelae Lauff. Iberodorcadion (Hispanodorcadion) becerrae (Lauffer, 1901) [Vives, 2000]. 
Obs. Nombre no disponible (CINZ Art.45.5, 45.6.2. Ver aberrantipes).

humerale. Dorcadion hispanicum v. cebollerense a. humerale Lauffer, 1911b: 41. Alrededores de Somosierra (Madrid). $\mathrm{N}^{\mathrm{o}}$ Cat. 8805.

Serie tipo: 1 ej.- SINTIPO $\sigma^{7}$. Somosierra, Lauffer $+D$. hisp. cebollerense ab. humerale Lauff. + ab. humerale Lauff. Sintipo. Iberodorcadion (Hispanodorcadion) perezi ssp. hispanicum (Mulsant, 1851) [Hernández, 2000]

Obs. Nombre no disponible (CINZ Art.45.5, 45.6.2. Ver aberrantipes).

ignotum. D. ignotum Escalera, 1911: 80-81. Teruel. No Cat. 8744.

Serie tipo: 55 ejs.- 2 SINTIPOS $\sigma^{x} \sigma^{x}$ : Teruel, A. Sanz + Dorcadion ignotum Esc. + I. (Hispanodorc.) zarcoi turdetanum (Esc.) E. Vives det. 1976. 7 SINTIPOS $\left(40^{7} \sigma^{7}, 3\right.$ 우 우): Teruel, A. Sanz + Dorcadion ignotum Esc. 46 SINTIPOS $\left(250^{7} \sigma^{\top}\right.$ y 21 우 우): Teruel, A. Sanz. Iberodorcadion (Hispanodorcadion) zarcoi ssp. turdetanum (Lauffer, 1911) [Vives, 1983].

incallosum. D. incallosum Escalera, 1908: 335. Cuenca del Riaza. $N^{\circ}$ Cat. 8755.

Serie tipo: 4 ejs.- LECTOTIPO $\sigma^{x}: 52 / 1+$ R. Riaza $+D$. incallosum Esc. + hololectotipo + Ib. (Hispanod.) graellsi ssp. incallosum E. Vives det. 1977. 3 PARALECTOTIPOS (10", 2 우 우): R. Riaza $+D$. incallosum Esc. + paralectotipo $+I b$. (Hispanod.) graellsi ssp. incallosum E. Vives det. 1977 [con etiqueta de numeración 52/2, 52/5-52/6]. Iberodorcadion (Hispanodorcadion) graellsii ssp. incallosum (Escalera, 1908) [Vives, 1983].

incanum. Dorcadion demandense a. incanum Lauffer, 1911a: 28-29. Barbadillo (Burgos). $\mathrm{N}^{\circ}$ Cat. 8821.

Serie tipo: 3 ejs.- SINTIPO $\sigma^{7}: D$. demandense a. incanum Lauff. + Sierra de Burgos $+D$. demandense a. incanum Lauff. Sintipo. 2 SINTIPOS $\sigma^{7} \sigma^{7}: D$. demandense a. incanum Lauff. Sintipo. Iberodorcadion (Hispanodorcadion) albicans ssp. demandense (Escalera, 1902) [Vives, 1983].

Obs. Nombre no disponible (CINZ Art.45.5, 45.6.2. Ver aberrantipes).

intermedium. D. Seguntianum v. intermedium Escalera, 1902: 287-288. Arroyo de Fraguas. $\mathrm{N}^{\circ}$ Cat. 8767.

Serie tipo: 2 ejs.- SINTIPO $\sigma^{n}$ : Arroyo Fraguas, J. Ardois + sintipo [en reverso: D. seguntianum var. intermedium Esc.1902]. SINTIPO $\sigma^{7}$ : Arroyo Fraguas, J. Ardois + sintipo [en reverso: $D$. seguntianum var. intermedium Esc.1902] + MNCN MADRID + Ib. (Hisp.) graellsi ssp. cinereum Lauffer E. Vives det. 1976. Iberodorcadion (Hispanodorcadion) seguntianum ssp. intermedium (Escalera, 1902) [Saz, 2010].

intuberculatum. Dorcadion Martinezi a. intuberculatum Lauffer, 1911a: 32. Madrid. Nº Cat. 8812.

Serie tipo: 1 ej.- SINTIPO $\sigma^{7}:$ Dorcadion martinezi a. intuberculatum + Retiro $+D$. martinezi ab. intuberculatum Lauff. Sintipo. Iberodorcadion (Hispanodorcadion) martinezii (Pérez Arcas, 1874) [Saz, 2007b].

Obs. Nombre no disponible (CINZ Art.45.5, 45.6.2. Ver aberrantipes). isernii. Dorcadion Isernii Pérez Arcas, 1868: 81. Somosierra. $\mathrm{N}^{\circ}$ Cat. 8800.

Serie tipo: 2 ejs.- LECTOTIPO $\sigma^{x}: 15 / 2+$ Dorcadion iserni Per. Somosierra [en reverso: Isern] + Col. Sr. Pérez Arcas + lectotipo + D. iserni P. A. O Escalera vidit + Iber. (Baeticodorcadion) iserni (Per. A.) E. Vives det. $1976+$ Lectotipo [en reverso: Vives desig. 1983] + MNCN Madrid. PARALECTOTIPO $\sigma^{x}: 15 / 1+$ Somosierra + Col. Sr. Pérez Arcas + paralectótipo + Iber. (Baeticodorcadion) iserni (Per. A.) E. Vives det 1976. Iberodorcadion (Baeticodorcadion) isernii (Pérez Arcas, 1868) [Vives, 1983].

lacunosum. D. lacunosum Escalera, 1908: 335. Cuenca del Záncara. $\mathrm{N}^{\circ}$ Cat. 8769.

Serie tipo: 3 ejs.- 2 SINTIPOS ( $\sigma^{\pi}$, ㅇ ): R. Zancara $+D$. panteli lacunosum Esc. + Sintipo D. lacunosum Esc. [con etiquetas de numeración: 54/1, 54/4]. SINTIPO $\sigma^{x}: 54 / 3+\mathrm{R}$. Zancara + Iber. (Hispanodorc.) uhagoni (P.A.) E. Vives det. 1977 + Sintipo D. lacunosum Esc. Iberodorcadion (Hispanodorcadion) uhagonii (Pérez Arcas, 1868). [Vives, 1983]

laufferi. Dorcadion Laufferi Escalera, 1900: 236-237. Cercedilla. No Cat. 8780.

Serie tipo: 16 ejs.- 2 SINTIPOS $\sigma^{x} \sigma^{x}$ : Cercedilla, J. Lauffer + Dorcadion laufferi Escal. 14 SINTIPOS $\left(90^{\prime \prime} \sigma^{\prime \prime}\right.$, 5 우 우): Cercedilla, J. Lauffer. Iberodorcadion (Hispanodorcadion) ghilianii ssp. cercedillanum (Pic, 1900) [Vives, 2000].

Obs. Nombre de taxon no válido por tratarse de un homónimo secundario del congenérico laufferi Pic, 1900 (CINZ, Art. 59.1). Por otra parte, los ejemplares de El Escorial anteriormente designados como lectotipo y paralectotipos (Vives, 1983) no corresponden a la serie original cuya localidad tipo es Cercedilla.

laufferoide. D. umbripenne var. laufferoide Escalera, 1911: 83. [En la descripción original no se indica localidad]. No Cat. 8775 .

Serie tipo: 17 ejs.- SINTIPO $\sigma^{\pi}$ : Pto. de Chia + umbripenne Esc. v. laufferoide Esc. + D. umbripenne var. laufferoide Esc. Sintipo. SINTIPO ㅇ: D. umbripenne v. laufferoide + D. umbripenne var. laufferoide Esc. Sintipo. 6 SINTIPOS $\sigma^{\top} \sigma^{\pi}$ : Villatoro, J. Ardois + D. umbripenne var. laufferoide Esc. Sintipo. 2 SINTIPOS 우 우: Villatoro, J. Ardois + circulito blanco. 7 SINTIPOS (5 $\sigma^{\top} \sigma^{\pi}, 2$ 우 우): Pto. Villatoro $+D$. umbripenne v. laufferoide Esc. Sintipo. Iberodorcadion (Hispanodorcadion) abulense (Lauffer, 1902) [Vives, 2000].

laurae. Iberodorcadion (s. str.) seoanei ssp. laurae Bahillo, 1994 [1993]: 65-69. Granucillo de Vidriales, Brime de Urz, Quiruelas de Vidriales (Zamora). N ${ }^{\circ}$ Cat. 11032.

Serie tipo: 4 ejs.- PARATIPOS $\left(20^{\pi} \sigma^{7}, 2\right.$ 우 우): Granucillo de Vidriales Za. UTM5759, 26-IV.1992, P. Bahillo leg. + paratipo + Iberodorcadion (s. str.) seoanei ssp. laurae P. Bahillo, 1993. Iberodorcadion (Iberodorcadion) seoanei $\mathbf{s s p}$. laurae Bahillo, 1994 [1993] [Bahillo, 1993].

loarrense. Iberodorcadion loarrense Berger, 1997: 39-48. Loarre, Huesca. $N^{\circ}$ Cat. 7459.

Serie tipo: 2 ejs.- HOLOTIPO $\sigma^{7}$ : Loarre, Huesca, 10.IV.1982, R. Gimeno leg. + $\sigma^{7}+$ holotype Iberodorcadion loarrensis P. Berger det. 1997. PARATIPO $Q$ : Loarre, sous le château, 15.III.1990, Huesca E. P. Berger leg. + + + allotype 
Iberodorcadion loarrensis P. Berger det. 1997. Iberodorcadion (Iberodorcadion) fuliginator ssp. loarrense (Berger, 1997) [González et al., 2007].

loroi. D. Loroi Escalera, 1924: 196-197. Soria. No Cat. 8736. Serie tipo: 7 ejs.- 2 SINTIPOS $\sigma^{x} \sigma^{x}$ : Soria, Loro $+D$. loroi Esc. sintipo. 3 SINTIPOS $\left(2 \sigma^{7} \sigma^{\pi}, 1\right.$ 우 $)$ : Soria, Loro $+D$. loroi Esc. sintipo + MNCN MADRID + Iberodorcadion becerrae Lauffer M. Tomé det. 1998. SINTIPO $\$$ : Soria, Loro + D. loroi Esc. sintipo + Iber. (Hispanodorc.) seguntianum becerrae v. loroi (Esc.) E. Vives det. 1977. SINTIPO ㅇ: Alrededores de Soria (Sr. Loro) + D. loroi Esc. sintipo + MNCN MADRID + Iberodorcadion becerrae Lauffer M. Tomé det. 1998. Iberodorcadion (Hispanodorcadion) becerrae (Lauffer, 1901) [Tomé, 1998].

marinae. Iberodorcadion (Hispanodorcadion) marinae Tomé \& Bahillo, 1996: 715-721. Burgos (España). N Cat. 12209.

Serie tipo: 8 ejs.- HOLOTIPO $0^{x}:$ Gen. $n^{\circ} 524+$ Olmillos de Sasamón, Burgos, 08.04.1996, P. Bahillo leg. + Holotipo Iberodorcadion marinae M. Tomé \& P. Bahillo det. 1998. PARATIPO $\sigma^{7}$ : Olmillos de Sasamón, Burgos, 07.04.1996, P. Bahillo leg. + paratipo Iberodorcadion marinae P. Bahillo det. 1996. 6 PARATIPOS ( $3 \sigma^{\top} \sigma^{\top}, 3$ 우 우): Olmillos de Sasamón, Burgos, 12.04.1996. P. Bahillo leg. + paratipo Iberodorcadion marinae P. Bahillo det. 1996. Iberodorcadion (Hispanodorcadion) albicans ssp marinae (Tomé y Bahillo, 1996) [Vives, 2000].

marmottani. Dorcadion Marmottani Escalera, 1900: 237-238. La Sagra (Granada), Sierra de Cabras (Murcia). No Cat. 8796.

Serie tipo: 10 ejs.- LECTOTIPO $\sigma^{2}: 13 / 3$ + La Sagra (Granada) Escalera $1900+$ D. marmottani Esc. $\sigma^{\top}$ tipo + lectotipo [en reverso: Vives desig. 1983] + I. (Baeticodorcadion) marmottani (Esc.) E. Vives det. 1976. PARALECTOTIPO $\sigma^{7}$ : Marmottani Escalera Typo [en reverso: La Sagra, Granada, 7, 900]. PARALECTOTIPO $\sigma^{7}:$ D. marmottani Esc. + marmottani typo. 2 PARALECTOTIPOS $\sigma^{\pi} \sigma^{\pi}$ : La Sagra (Granada), Escalera, $1900+$ D. marmottani Esc. $\sigma^{7}$ tipo + paralectotipo + I. (Baeticodorcadion) marmottani (Esc.) E. Vives det. 1976 [con etiquetas de numeración: 13/1, 13/2]. 3 PARALECTOTIPOS $\bigcirc \div$ : La Sagra (Granada) Escalera $1900+D$. marmottani Esc. O tipo + paralectotipo $+I$. (Baeticodorcadion) marmottani (Esc.) E. Vives det. 1976. [con etiquetas de numeración: 13/5, 13/6 y 13/7]. PARALECTOTIPO O : 13/4 + La Sagra (Granada) Escalera $1900+$ D. marmottani Esc. $O^{7}$ tipo + I. (Baeticodorcadion) marmottani ssp. cabrasense (Nic.) E. Vives det. $1976+$ D. marmottani Esc. Paralectotipo. PARALECTOTIPO $\circ: 13 / 8+$ La Sagra (Granada) Escalera $1900+$ D. marmottani Esc. $ᄋ$ tipo $+D$. marmottani Esc. Paralectotipo. Iberodorcadion (Baeticodorcadion) marmottani (Escalera, 1900) [Vives, 1983].

martinezii. Dorcadion Martinezii Pérez Arcas, 1874: 145. Madrid. $\mathrm{N}^{\mathrm{o}}$ Cat. 8765.

Serie tipo: 3 ejs.- LECTOTIPO $\sigma^{7}: 44 / 1+$ Madrid + ejem. dibujado + D. martinezi P. A. $\sigma^{7}$ Esc. Vidit + col. Sr. Pérez Arcas + lectotipo + Iber. (Hispanodoc.) martinezi (P.A.) E. Vives de. 1977. PARALECTOTIPO $\sigma^{7} .44 / 3+$ Madrid $+D$. martinezi P. A. $\sigma^{\pi}$ Esc. Vidit + col. Sr. Pérez Arcas + paralectotipo. PARALECTOTIPO $\sigma^{\pi}: 44 / 4+$ Dorcadion 3, Madrid + D. martinezi P. A. $\sigma^{7}$ Esc. vidit + col. Sr. Pérez Arcas + para- lectotipo + Iber. (Hispanodorc.) martinezi (P. A.) E. Vives det. 1977. Iberodorcadion (Hispanodorcadion) martinezii (Pérez Arcas, 1874) [Vives, 1983].

montanum. Dorcadion Dejeani var. montanum Lauffer, 1911b: 53-54. Montañas altas de Sierra de Béjar. N ${ }^{\text {C }}$ Cat. 8806.

Serie tipo: 11 ejs.- SINTIPO $\sigma^{7}$ : Sierra de Béjar, 6/1905, J. Lauffer $+D$. dejeani montanum Lauf. $+D$. dejeani var. mon-

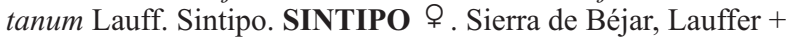
Ib. (Hispanodorc.) segovianum ssp. dejeani (Chevr.) E. Vives det. 1977. SINTIPO $\sigma^{7}$ : Sierra de Béjar, Lauffer +896 (etiq. roja) + Dorcadion Dejeani. 7 SINTIPOS $\left(30^{\pi} \sigma^{\pi}, 4\right.$ 우 우): Sierra de Bejar, Lauffer. SINTIPO $\sigma^{7}: D$. dejeani montanum Lauffer. Iberodorcadion (Hispanodorcadion) segovianum ssp. dejeanii (Chevrolat, 1862) [Vives, 2000].

mosqueruelense. D. Mosqueruelense Escalera, 1902: 283-284. Zaragoza, Mosqueruela (Teruel). $\mathrm{N}^{\circ}$ Cat. 8735.

Serie tipo: 6 ejs.- LECTOTIPO $\sigma^{7}: 29 / 4+$ Mosqueruela + D. mosqueruelense $\sigma^{7}$ tipo + lectotipo $+I b$. (Hisp.) mosqueruelense Esc., E. Vives det. $1977+$ MNCN MADRID + I. (Hisp.) pseudomolitor (Esc. 1902) González det. 2002. 2 PARALECTOTIPOS $\sigma^{7} \sigma^{7}$ : Mosqueruela $+D$. mosqueruelense Esc. paralectotipo + Ib. (Hisp.) mosqueruelense Esc., E. Vives det. $1977+$ MNCN MADRID + I. (Hisp.) pseudomolitor (Esc. 1902) González det. 2002 [con etiquetas de numeración: 29/1,29/2]. PARALECTOTIPO $\sigma^{\pi}:$ 29/3 + Mosqueruela $+D$. mosqueruelense $\sigma^{7}$ tipo + paralectotipo + Ib. (Hispanodorcadion) mosqueruelense E. Vives det. $1977+$ I. (Hisp.) pseudomolitor (Esc. 1902) González det. 2002. PARALECTOTIPO $ᄋ: 29 / 5+$ Mosqueruela + D. mosqueruelense ㅇ tipo + paralectotipo $+I b$. (Hisp.) mosqueruelense Esc., E. Vives det. $1977+$ MNCN MADRID + I. (Hisp.) pseudomolitor (Esc. 1902) González det. 2002. PARALECTOTIPO $\sigma^{x}$ : Mosqueruela $(\mathrm{Te})+$ paralectotipo $+I b$. (Hispanodorcadion) mosqueruelense E. Vives det. $1977+$ Iberodorcadion mosqueruelense Escalera M. + I. (Hisp.) pseudomolitor (Esc. 1902) González det. 2002. Iberodorcadion (Hispanodorcadion) mosqueruelense (Escalera, 1902) [Vives, 1983].

navasi. Dorcadion Navasi Escalera, 1900: 234-235. Zaragoza. $\mathrm{N}^{\circ}$ Cat. 8760.

Serie tipo: 1 ej.- NEOTIPO $\sigma^{\pi}: 31 / 1+$ Dorcadion navasi Esc. (Zaragoza) 29 marzo $1902+$ D. Navasi Esc. $\sigma^{\top}$ Escalera vidit + neoholotipo $+I b$. (Hisp.) molitor navasi (Esc.) E. Vives det. 1977. Iberodorcadion (Hispanodorcadion) molitor ssp. navasi (Escalera, 1900) [Vives, 1983].

neilense. Dorcacion neilense Escalera, 1902: 278-279. Neila (Burgos). $\mathrm{N}^{\mathrm{o}}$ Cat. 8751.

Serie tipo: 3 ejs.- LECTOTIPO $\sigma^{\pi}: 32 / 1+$ Neila + D. neilense Esc. $\sigma^{\prime \prime}$ tipo + holotipo $+I b$. (Hisp.) neilense (Esc.) E. Vives det $1977+$ MNCM Cat. Tipos $n^{\circ} 8751$. PARALECTOTIPO $\sigma^{7}:$ 32/2 + Canales Pr. Logroño, Spain G.C.C. + D. neilense $\mathrm{R}^{\circ}$ de Campion $+D$. neilense Esc. $\sigma^{\top}$ Escalera vidit + paratipo. PARALECTOTIPO $\sigma^{n}$ : Neila $+D$. neilense + paratipo + Ib. (Hisp.) neilense (Esc.) E. Vives det. $1977+$ MNCN Cat. Tipos $\mathrm{M}^{\circ}$ 8751. Iberodorcadion (Hispanodorcadion) neilense (Escalera, 1902) [Vives, 1983].

Obs. La designación de Holotipo (Vives, 1983) basada en la indicación tipo, presente en el etiquetado y a mano del autor, 
ha de interpretarse como una designación de Lectotipo (CINZ Art. 73.1, Recom. 73F, Art. 74.6).

nigratum. D. villosladense var. nigratum Escalera, 1924: 200. Cameros. $\mathrm{N}^{\circ}$ Cat. 8788.

Serie tipo: 6 ejs.- 5 SINTIPOS $\sigma^{\pi} \sigma^{x}$ : Cameros, C. Bolívar + D. villosladense v. nigratum Esc. Sintipo + MNCN Cat. Tipos N $\mathrm{N}^{0}$ 8788. SINTIPO $\sigma^{7}$ : Cameros, C. Bolívar $+I b$. (Hispan.) neilense nicolasianum E. Vives det. $1977+$ D. villosladense v. nigratum Esc. Sintipo + MNCN Cat. Tipos No 8788. Iberodorcadion (Hispanodorcadion) neilense (Escalera, 1902) [Vives, 2000).

nigrinum. D. palentinum var. nigrinum. Escalera, 1911: 82. Salinas del Pisuerga. N ${ }^{\circ}$ Cat. 8823.

Serie tipo: 1 ej.- SINTIPO $\sigma^{x}$ : Salinas de Pisuerga, Palencia, Cendrero $+D$. palentinum Esc. v. nigrinum Esc. + Iber. (Hisp.) albicans ssp. palentinum (Esc.) E. Vives det. $+D$. palentinum v. nigrinum Esc. Sintipo. Iberodorcadion (Hispanodorcadion) albicans ssp. palentinum (Lauffer, 1911) [Vives, 2000].

nigripenne. D. umbripenne var. nigripenne Escalera, 1911: 8283. [En la descripción original no se indica localidad]. $\mathrm{N}^{\circ}$ Cat. 8776.

Serie tipo: 9 ejs.- SINTIPO 으 : Valle del Tormes $+I b$. (Hisp.) abulense (Lauff.) E. Vives det. $1977+$ D. umbripenne v. nigripenne Sintipo. 6 SINTIPOS (5 $\sigma^{7} \sigma^{7}, 1$ 우): Pto. de Chía $+D$. umbripenne v. nigripenne Esc. Sintipo. 2 SINTIPOS $\sigma^{x} \sigma^{x}$ : Valle del Tormes $+D$. umbripenne v. nigripenne Sintipo. Iberodorcadion (Hispanodorcadion) abulense (Lauffer, 1902) [Vives, 2000].

nigritulus. D. pseudomolitor var. nigritulus Escalera, 1924: 197-198. Peña Golosa. N ${ }^{\circ}$ Cat. 8738.

Serie tipo: 2 ejs.- 2 SINTIPOS ( $\sigma^{\top}$, Q ): Peña Golosa, Cs. + pseudomolitor v. nigritulus sintipo $+I$. (Hispanodorcadion) mosqueruelense carbonarium (Nic.) E. Vives det. 1976. Iberodorcadion (Hispanodorcadion) pseudomolitor (Escalera, 1902) [Saz, 2009].

nigrosuturale. Dorcadion Perezi a. nigrosuturale Lauffer, 1911c: 74. Alrededores del Escorial. No Cat. 2334.

Serie tipo: 1 ej.- SINTIPO $\sigma^{7}$ : Escorial, Lauffer $+D$. pere$z i$ f. transitoria a ghilianii. Iberodorcadion (Hispanodorcadion) perezi (Graells, 1849) [Vives, 2000].

Obs. Nombre no disponible (CINZ Art. 45.5, 45.6.2. Ver aberrantipes).

nivosum. D. villosladense var. nivosus Escalera, 1924: 198199. Cameros. No Cat. 8778.

Serie tipo: 3 ejs.- SINTIPO $\sigma^{x}$ : Cameros, C. Bolivar $+C$. villosladense v. nivosus Esc. Sintipo $+\mathrm{MNCN}$ Cat. Tipos $\mathrm{N}^{\circ}$ 8778. SINTIPO $\sigma^{\circ}$ : Cameros, C. Bolivar $+I b$. (Hisp.) neilense nivosum Vives det. $1977+$ D. villosladense v. nivosus Esc. Sintipo + MNCN Cat. Tipos No 8778. SINTIPO 우: Cameros, C. Bolivar $+I b$. (Hisp.) neilense vicenti Vives det. $1977+D$. villosladense v. nivosus Esc. Sintipo + MNCN Cat. Tipos $N^{\circ}$ 8778. Iberodorcadion (Hispanodorcadion) neilense (Escalera, 1902) [Vives, 2000].

Obs. Lapsus calami del autor. nudipenne. D. nudipenne Escalera, 1908: 337. Cuenca del Riaza. $\mathrm{N}^{\circ}$ Cat. 8781.

Serie tipo: 31 ejs.- LECTOTIPO 9 : Fresno + D. nudipenne Esc. + lectotipo + Iber. (Hispanodorc.) ssp. nudipenne (Esc.) E. Vives det 1977. PARALECTOTIPO $\sigma^{7}$ Fresno. 14 PARALECTOTIPOS (11 $0^{7} \sigma^{7}, 3$ 우 우): Riaza [dos ejemplares con etiqueta de numeración: 64/6, 64/7]. 2 PARALECTOTIPOS $\left(\sigma^{\prime}, \circ\right)$ : Riaza, J. Ardois. 3 PARALECTOTIPOS

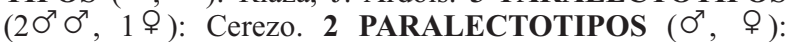
Cerezo, J. Ardois. 2 PARALECTOTIPOS ( $\sigma^{\prime \prime}$,, $)$ : Cerezo de Arriba (Segovia) + Cerezo, J. Ardois. 4 PARALECTOTIPOS $\left(3 \sigma^{\pi} \sigma^{\pi}, 1\right.$ ) $)$ : Cerezo de Arriba (Segovia) [dos ejemplares con etiqueta de numeración: 64/2, 64/4]. 2 PARALECTOTIPOS $\left(\sigma^{7}\right.$, + ): Somosierra. Iberodorcadion (Hispanodorcadion) perezi ssp. nudipenne (Escalera, 1908) [Saz, 2011b].

obscuratum. D. villosladense var. obscuratus Escalera, 1924: 199. Villoslada, Cameros. No Cat. 8786

Serie tipo: 26 ejs.- SINTIPO 울 Cameros, C. Bolivar + villosladense $+D$. villosladense v. obscuratus Sintipo + MNCN Cat. Tipos $\mathrm{N}^{\circ}$ 8786. SINTIPO 우: Cameros, C. Bolivar $+I b$. (H.) neilense obscuratus E. Vives det. $1977+D$. villosladense v. obscuratus Sintipo $+\mathrm{MNCN}$ Cat. Tipos $\mathrm{N}^{\mathrm{o}}$ 8786. 24 SINTIPOS (20 O $0^{x}, 4$ 우 우 ): Cameros, C. Bolivar + D. villosladense v. obscuratus Sintipo + MNCN Cat. Tipos $\mathrm{N}^{\mathrm{o}}$ 8786. Iberodorcadion (Hispanodorcadion) neilense (Escalera, 1902) [Vives, 2000].

Obs. Lapsus calami del autor.

occidentalis. D. Graellsi var. occidentalis Escalera, 1924: 192. Puerto de Villatoro, Puerto de Chía. No Cat. 2342.

Serie tipo: 31 ejs.- SINTIPO $\sigma^{x}$ : Pto. de Chia + graellsi v. occidentalis Esc. SINTIPO $\sigma^{\pi}$. Pto. de Villatoro + graellsi $\mathrm{v}$. occidentalis Esc. 23 SINTIPOS (140 $\sigma^{x}, 9$ 9 $ᄋ$ ) Pto. de Villatoro. SINTIPO $ᄋ$ : Pto. de Villatoro + Ib. (Hisp.) segovianum E. Vives det. 1977. 5 SINTIPOS (3 $\sigma^{x} \sigma^{\pi}, 2$ ㅇ ) ): Pto. de Chia. Iberodorcadion (Hispanodorcadion) graellsii (Graells, 1858).

Obs. Nombre no disponible, por carecer de descripción (CINZ Art. 12.1). Enumerado por Escalera, pero no descrito. Los 31 sintipos son atribuibles, en opinión de los autores, a la especie I. (H.) graellsii.

palentinum. Dorcadion palentinum Lauffer, 1911a: 27-28. Salinas de Pisuerga. N ${ }^{\circ}$ Cat. 8822.

Serie tipo: 17 ejs.- LECTOTIPO $\sigma^{\pi}$ : Salinas de Pisuerga, Palencia, Cendrero + D. palentinum Esc. + lectotipo + Iber (Hispanodorc.) albicans ssp. palentinum (Esc.) E. Vives det. 11 PARALECTOTIPOS (7 $0^{7} 0^{7}, 4$ 우 우): Salinas de Pisuerga, Palencia, Cendrero $+D$. palentinum Esc. + paralectotipo. 4 PARALECTOTIPOS $\left(3 \sigma^{\pi} \sigma^{7}, 1\right.$ 웅: Salinas de Pisuerga, Palencia, Cendrero + paralectotipo + Iber. (Hispanodorc.) albicans ssp. palentinum (Esc.) E. Vives det. PARALECTOTIPO $\sigma^{\top}$. Salinas de Pisuerga, Palencia, Cendrero + paralectotipo. Iberodorcadion (Hispanodorcadion) albicans ssp. palentinum (Lauffer, 1911) [Vives, 2000].

paradoxum. D. paradoxum Escalera, 1908: 336. Cuenca del Cega. No Cat. 8757.

Serie tipo: 6 ejs.- 3 SINTIPOS $\left(2 \sigma^{7} \sigma^{\top}, 1\right.$ 우 $)$ : Iscar $+D$. paradoxum Esc. [el ejemplar 우 con etiqueta de numeración: 55/3]. SINTIPO $\sigma^{7}: 55 / 1+$ R. Cega + D. paradoxum Esc. + 
Sintipo D. paradoxum Esc. + MNCN MADRID. SINTIPO 우 : $55 / 2+$ R. Cega + D. paradoxum Esc. + Sintipo D. paradoxum Esc. SINTIPO $\sigma^{7}: 55 / 4+D$. paradoxum Esc. Iberodorcadion (Hispanodorcadion) graellsii ssp. incallosum (Escalera, 1908) [Vives, 2000].

parmeniforme. D. parmeniforme Escalera, 1902: 288-289. Santiago de la Espada (Granada). N ${ }^{\circ}$ Cat. 8750.

Serie tipo: 2 ejs.- 2 SINTIPOS ( $\sigma^{\pi}$, 우): Santiago de la Espada $+D$. parmeniforme Esc. tipo + Sintipo D. parmeniforme Esc. + Ib. Baeticodorcadion mucidum Dalman, 1817. Zapata det. 1998. [los ejemplares con etiqueta de numeración: 4/1, 4/2]. Iberodorcadion (Baeticodorcadion) mucidum (Dalman, 1817). [Verdugo, 2003]

paulae. Iberodorcadion (Hispanodorcadion) paulae Corraleño \& Murria, 2012: 43-55. Jaulín (Zaragoza). $\mathrm{N}^{\mathrm{o}}$ Cat. 2211.

Serie tipo: 2 ejs.- 2 PARATIPOS (O", \& ): Jaulín (Z.), 26III-2011; A. Murria leg. + Iberodorcadion (Hispanodorcadion) paulae n. sp., Corraleño \& Murria det. + MNCN, Cat. Tipos 2211. Iberodorcadion (Hispanodorcadion) paulae Corraleño \& Murria, 2012

penascosum. Dorcadion Amori v. peñascosum Lauffer, 1911b: 44-45. Peñascosa, $S^{a}$ de Alcaraz (Albacete), Ruidera (Ciudad Real). $N^{\circ}$ Cat. 8817.

Serie tipo: 8 ejs.- SINTIPO 9 : Sierra de Alcaraz, 29/1/03 + D. amori peñascosum Lauff. $+D$. amori var. peñascosum Lauff. Sintipo. 5 SINTIPOS 우 우: Sierra de Alcaraz, 29/1/03. SINTIPO $\sigma^{7}: D$. amori v. peñascosum Lauff. SINTIPO $\sigma^{\pi}$ : Sierra de Alcaraz + MNCN MADRID. Iberodorcadion (Baeticodorcadion) amorii (Marseuil, 1856) [Vives, 2000].

perezi. Dorcadion perezi Graells, 1849: 5. Montaña media de Guadarrama. No Cat. 8763.

Serie tipo: 1 ej.- HOLOTIPO $\sigma^{x}$ : Gredos, Escorial + Dorcadion perezi + holotipo + Iberodorcadion (Hispanodorcadion) perezi (Graells) E. Vives det. 1977. Iberodorcadion (Hispanodorcadion) perezi (Graells, 1849) [Vives, 1983].

Obs. Error en el etiquetado del ejemplar: Gredos por Guadarrama.

perezoide. D. umbripenne var. Perezoide Escalera, 1911: 84. [En la descripción original no se indica localidad]. $\mathrm{N}^{\mathrm{o}}$ Cat. 8773.

Serie tipo: 3 ejs.- SINTIPO \& : Pto. Chia $+D$. umbripenne v. perezoide Esc. Sintipo. SINTIPO 우: Menga, J. Ardois + D. umbripenne v. perezoide Esc. Sintipo. SINTIPO 우: Pto. Villatoro + D. umbripenne v. perezoide Esc. Sintipo. Iberodorcadion (Hispanodorcadion) abulense (Lauffer, 1902) [Vives, 2000].

plurilineatum. D. umbripenne var. plurilineatum Escalera, 1911: 84. [En la descripción original no se indica localidad]. $N^{\circ}$ Cat. 8772.

Serie tipo: 9 ejs.- 3 SINTIPOS 우 우: Menga, J. Ardois + D. umbripenne v. plurilineatum. Sintipo. SINTIPO $\bigcirc$ : Villatoro, J. Ardois + Ib. (Hisp.) abulense (Lauff.) E. Vives det. $1977+$ D. umbripenne v. plurilineatum Sintipo. 5 SINTIPOS $\left(20^{\top} \sigma^{\pi}, 3\right.$ 우 우): Villatoro, J. Ardois + D. umbripenne v. plurilineatum Sintipo. Iberodorcadion (Hispanodorcadion) abulense (Lauffer, 1902) [Vives, 2000]. pradae. Iberodorcadion (Hispanodorcadion) uhagonii pradae Saz, 2007a: 195-199. Sierra Ministra (Soria). No Cat. 9913.

Serie tipo: 2 ejs.- HOLOTIPO $\sigma^{x}: 15-V I-1997$, ex larva, Srra. Ministra, Soria, Del Saz \& López Prada leg. + holotypus Ib. (H.) uhagonii pradae ssp n. Del Saz des. $2006+\mathrm{MNCN}$ Cat. Tipos N $^{o}$ 9913. PARATIPO $\sigma^{x}$ : 24-VI-1997, ex larva, Srra. Ministra, Soria, Del Saz \& López Prada leg. + alotypus Ib. (H.) uhagonii pradae ssp n. Del Saz des. $2006+$ MNCN Cat. Tipos $\mathrm{N}^{\circ}$ 9913. Iberodorcadion (Hispanodorcadion) uhagonii ssp. pradae Saz, 2007. [Saz, 2007a].

pruinosum. D. pruinosum Escalera, 1902: 285-286. Alcuneza, Matillas, Sigüenza, Cuenca y Burgos. № Cat. 8756.

Serie tipo: 5 ejs.- 2 SINTIPOS $\left(\sigma^{7}\right.$, ㅇ ): Alcuneza $+D$. pruinosum Esc. sintipo + MNCN MADRID + Iberodorcadion becerrae Lauffer M. Tomé det. 1998 [con etiquetas de numeración: 42/1, 42/2]. SINTIPO $\sigma^{7}$ : Sigüenza, J. Ardois $+D$. pruinosum Esc. $\sigma^{7}$ tipo $+I b$. Hispanodorc. seguntianum v. pruinosum E. Vives det. 1977. SINTIPO $\sigma^{7}$ : Sigüenza, J. Ardois + MNCN + Iberodorcadion becerrae Lauffer Mario Tomé det. 1998. SINTIPO 우 Sigüenza J. Ardois. Iberodorcadion (Hispanodorcadion) becerrae (Lauffer, 1901) [Tomé, 1997].

pseudomolitor. D. Mosqueruelense Var. pseudo-molitor Escalera, 1902: 285. Peña Golosa (Castellón). № Cat. 8737.

Serie tipo: 2 ejs.- 2 SINTIPOS ( $\sigma^{\top}$, $ᄋ$ ): Peña Golosa $+D$. mosqueruelense v. pseudomolitor Esc. sintipo $+I$. (Hispanodorcadion) mosqueruelense (Esc.) E. Vives det. 1976. Iberodorcadion (Hispanodorcadion) pseudomolitor (Escalera, 1902) [González et al., 2001].

pulvipenne. D. pulvipenne Escalera, 1908: 335. Cuenca del Duratón. No Cat. 8766.

Serie tipo: 4 ejs.- 3 SINTIPOS (20 $\sigma^{\pi}, 1$ \& ): R. Duratón + D. pulvipenne Esc. + Sintipo D. pulvipenne Esc. [con etiquetas de numeración: 53/1, 53/3, 53/4]. SINTIPO o : 53/2+R. Duratón + D. pulvipenne Esc. + Ib. (Hispanod.) graellsi ssp. incallosum E. Vives det. 1977 + Sintipo D. pulvipenne Esc. Iberodorcadion (Hispanodorcadion) graellsii ssp. incallosum (Escalera, 1908) [Vives, 2000].

rondense. Iberodorcadion mucidum rondensis Verdugo, 2003: 128-129. Sierra de las Nieves, Ronda (Málaga), Sierra de Grazalema (Cádiz). Nº Cat. 9359.

Serie tipo: 2 ejs.- HOLOTIPO $\sigma^{x}$ : Ronda, Málaga, 25 Mayo 1996, A. Verdugo leg. + Iberodorc. mucidum (Dalman, 1817) A. Verdugo det. 1996 + Holotypus Iberodorcadion (Baet.) mucidum (Dalman, 1817) ssp. rondensis nova $\sigma^{7} \mathrm{~A}$. Verdugo des. $2002+$ MNCN Cat. Tipos No 9359. PARATIPO Q : Ronda, Málaga, 25 Mayo 1996, A. Verdugo leg. + Iberodorc. mucidum (Dalman, 1817) A. Verdugo det. $1996+$ Allotypus Iberodorcadion (Baet.) mucidum (Dalman, 1817) ssp. rondensis nova $\bigcirc$ A. Verdugo des. $2002+\mathrm{MNCN}$ Cat. Tipos $\mathrm{N}^{\circ}$ 9359. Iberodorcadion (Baeticodorcadion) mucidum ssp. rondensis Verdugo, 2003. [Verdugo, 2003].

Obs. Lapsus calami del autor.

rufipedes. D. villosladense var. rufipedes Escalera, 1924: 199. Cameros. $N^{\circ}$ Cat. 8779.

Serie tipo: 4 ejs.- 3 SINTIPOS $\sigma^{\top} \sigma^{7}$ : Cameros, C.Bolivar + D.villosladense v.rufipedes Esc. Sintipo + MNCN Cat. Tipos $\mathrm{N}^{\mathrm{o}} 8779$. SINTIPO 우: Cameros, C. Bolivar $+I b$. (Hisp.) nei- 
lense villosladense Vives det. $1977+$ D. villosladense v. rufipedes Esc. Sintipo + MNCN Cat. Tipos No 8779. Iberodorcadion (Hispanodorcadion) neilense (Escalera, 1902) [Vives, 2000].

salmantinum. D. Salmantinum Escalera, 1924: 193. Salamanca. ). $\mathrm{N}^{\mathrm{o}}$ Cat. 2345.

Serie tipo: 1 ej.- SINTIPO $\sigma^{x}$ : Salamanca + Dor. (Hispanodorc.) paradoxum (Esc.) E. Vives det 1977. Iberodorcadion (Hispanodorcadion) graellsii (Graells, 1858).

Obs. Nombre no disponible, por carecer de descripción (CINZ Art. 12.1). Enumerado por Escalera, pero no descrito. El sintipo es atribuible, en opinión de los autores, a la especie I. (H.) graellsii.

sanzi. D. turdetanum a. Sanzi Lauffer, 1911a: 27. Teruel. No Cat. 8746.

Serie tipo: 1 ej.- SINTIPO $\sigma^{\pi}:$ D. turdetanum a. sanzi Lauff. + sintipo + Iber. (Hispanodorc.) zarcoi turdetanum Lauffer E. Vives det. $1977+$ MNCN MADRID. Iberodorcadion (Hispanodorcadion) zarcoi ssp. turdetanum (Lauffer, 1911) [Vives, 2000].

Obs. Nombre no disponible (CINZ Art. 45.5, 45.6.2. Ver aberrantipes).

saturioi. D. Saturioi Escalera, 1924: 198. Silos. № Cat. 8761.

Serie tipo: 3 ejs.- 2 SINTIPOS $\left(0^{x}\right.$, ㅇ $)$ : Silos + D. saturioi sintipo + MNCN MADRID + Iberodorcadion becerrae Lauffer, M. Tomé det. 1998. SINTIPO 우: Silos + D. saturioi sintipo + Ib. (Hisp.) seguntianum saturioi (Esc.) E. Vives det. 1977. Iberodorcadion (Hispanodorcadion) circumcinctum (Chevrolat, 1862) [Vives, 2000].

seeboldi. D. Heydeni v. Seeboldi Escalera, 1902: 288. Bilbao. $\mathrm{N}^{\circ}$ Cat. 8762.

Serie tipo: 7 ejs.- SINTIPO $\sigma^{7}: 37 / 1+$ Dorcadion 1 Bilbao [en reverso: Mieg] + col. Sr. Pérez Arcas + D. Seeboldi Esc. $\sigma^{7}$ tipo $+D$. heydeni var. seeboldi Esc. sintipo + MNCN MADRID. 2 SINTIPOS $\sigma^{7} \sigma^{7}$ : Bilbao, Mieg + col. Sr. Pérez Arcas $+D$. Seeboldi Esc. $\sigma^{7}$ tipo $+D$. heydeni var. seeboldi Esc. sintipo [con etiquetas de numeración: $37 / 2,37 / 3$ ]. 4 SINTIPOS $\left(2 \sigma^{7} \sigma^{7}\right.$, 2 우 우): Bilbao + coll. Seebold, Mus. Madrid + D. heydeni var. seeboldi Esc. sintipo [Uno de los ejemplares con etiqueta de numeración: 204]. Iberodorcadion (Hispanodorcadion) circumcinctum (Chevrolat, 1862) [Saz, 2011a]

seguranum. Dorcadion amori v. seguranum Lauffer, 1911b: 45. Sierra de Segura (Jaén). N ${ }^{\circ}$ Cat. 2336.

Serie tipo: 2 ejs.- SINTIPOS $\sigma^{7} \sigma^{x}: S^{a}$ Segura $+I b$. Baeticodorcadion amori ssp. segurense Escalera, 1911. Zapata det. 1999. Iberodorcadion (Baeticodorcadion) amorii ssp. segurense (Escalera, 1911) [Vives, 2000].

segurense. D. Segurense Escalera, 1911: 82. Mancha Alta. N Cat. 8799.

Serie tipo: 11 ejs.- LECTOTIPO $\sigma^{x}: 49 / 4+$ Masegoso $+D$. ruiderense Esc. $\sigma^{7}$ tipo + lectotipo $+I$. (Baeticodorcadion) amori ssp. segurense (Escalera) E. Vives det. $1977+$ lectotipo (en reverso: Vives desig. 1983). 2 PARALECTOTIPOS $\sigma^{x} \sigma^{7}$ : Alcaraz + D. ruiderense Esc. $\sigma^{7}$ tipo $+D$. segurense Esc. Paralectotipo [con etiquetas de numeración: 49/1, 49/2]. 3 PARALECTOTIPOS $\sigma^{7} \sigma^{7}$ : Masegoso + D. ruiderense Esc. $\sigma^{7}$ tipo $+D$. segurense Esc. Paralectotipo [con etiquetas de numeración: 49/3, 49/5, 49/11]. 2
PARALECTOTIPOS $ᄋ$ 울 Alcaraz $+D$. ruiderense Esc. 우 tipo $+D$. segurense Esc. Paralectotipo [con etiquetas de numeración: 49/8, 49/10]. PARALECTOTIPO $\sigma^{7}:$ 49/7 + Peñascosa + $D$. ruiderense Esc. $\sigma^{\pi}$ tipo $+D$. segurense Esc. Paralectotipo. PARALECTOTIPO $ᄋ: 49 / 9+$ Peñascosa $+D$. ruiderense Esc.

O tipo + paralectotipo. + I. (Baet.) amori segurense E. Vives. det. 1976. PARALECTOTIPO ㅇ: $49 / 12+$ Masegoso + D. ruiderense Esc. O tipo $+D$. segurense Esc. Paralectotipo. Iberodorcadion (Baeticodorcadion) amorii ssp. segurense (Escalera, 1911) [Vives, 1983].

seoanei. Dorcadion seoanei Graells, 1858: 93. Pto. del Manzanal (León). $\mathrm{N}^{\mathrm{o}}$ Cat. 8801.

Serie tipo: 2 ejs.- LECTOTIPO $\sigma^{x}: 11 / 1+$ Dorcadion seoanei Gr. P. Manzanal [en reverso: Seoane] + col. Sr. Pérez Arcas + lectótipo. PARALECTOTIPO $\sigma^{7}: 11 / 2$ + Puerto del Manzanal + col. Sr. Pérez Arcas + paralectótipo. Iberodorcadion (Iberodorcadion) seoanei (Graells, 1858) [Vives, 1983].

septemvittatum. Dorcadion Perezi $ᄋ$ var. septemvittatum Lauffer, 1901: 95-96. Sierra de Guadarrama, Escorial. No Cat. 2335.

Serie tipo: 1 ej.- SINTIPO 을 Escorial, J. Lauffer $+D$. pere$z i$ a. 7vittatum Lauffer. Iberodorcadion (Hispanodorcadion) perezi (Graells, 1849) [Vives, 2000].

steparium. Dorcadion steparius Escalera, 1900: 239-240. Villahermosa (Albacete), Gallocanta (Zaragoza), Uclés (Cuenca), Albarracín (Teruel). [La localidad Villahermosa corresponde a la provincia de Ciudad Real, no a Albacete]. $\mathrm{N}^{\circ}$ Cat. 8795 .

Serie tipo: 6 ejs.- SINTIPO $\sigma^{\pi}: 14 / 1+D$. lorquini Kraatz, Albarracín [en reverso: Zapater] + Col. Sr. Pérez Arcas $+D$. steparium Esc. $\sigma^{7}$ tipo + D. steparius Esc. Sintipo. SINTIPO o : 14/2 Albarracín + Col. Sr. Pérez Arcas $+D$. steparium Esc. o tipo + D. steparius Esc. Sintipo. SINTIPO 우: 14/3 + Dorcadion 10 Uclés [en reverso: Pantel] + Col. Sr. Pérez Arcas + D. steparium Esc. $ᄋ$ tipo + Iber. (Baet.) iserni (Per. Arc.) E. Vives det $1976+$ D. steparius Esc. Sintipo. SINTIPO ㅇ : 14/4 + Cerro Cortes Barranco, Arguido + D. steparius Esc. Sintipo. SINTIPO 우 : 14/5 + Virgen Carmen, Albarracín, 8 abril $+D$. steparius Esc. Sintipo. SINTIPO $\sigma^{x}: 14 / 6+$ Iber. (Baeticodorcadion) iserni (Per.A.) E. Vives det $1976+$ D. steparius Esc. Sintipo. [el ejemplar lleva una etiqueta rectangular amarilla]. Iberodorcadion (Baeticodorcadion) isernii (Pérez Arcas, 1868) [Vives, 2000].

Obs. Lapsus calami del autor

submontanum. Dorcadion Dejeani var. submontanum Lauffer, 1911b: 54-55. Candelario y sus alrededores. $\mathrm{N}^{\mathrm{o}}$ Cat. 8807.

Serie tipo: 5 ejs.- SINTIPO $\sigma^{7}$ : Candelario, $31.4+D$. dejeani v. submontanum Lauffer $+D$. dejeani submontanum Lauff. Sintipo. SINTIPO $\sigma^{7}$ : Candelario, $31.4+I b$. (Hispanodorc.) segovianum ssp. dejeani (Chevrl.) E. Vives det. $1977+$ D. dejeani submontanum Lauff. 2 SINTIPOS (O", ㅇ ): Candelario, $31.4+D$. dejeani submontanum Lauff. Sintipo. SINTIPO $\sigma^{\top}:$ D. dejeani submontanum Lauff. Iberodorcadion (Hispanodorcadion) segovianum ssp. dejeanii (Chevrolat, 1862) [Vives, 2000].

subpolitum. Dorcadion Laufferi $ᄋ$ 으. vabpolitum Lauffer, 1901: 98. Sierra Guadarrama, Garganta del Espinar. N Cat. 7720. 
Serie tipo: 1 ej.- SINTIPO 우: D. laufferi var. 우 subpolitum Lauff. + Cercedilla, J. Lauffer + sintipo D. laufferi v. subpolitum Lauff. Iberodorcadion (Hispanodorcadion) ghilianii ssp. cercedillanum (Pic, 1900) [Vives, 2000].

superbum. Dorcadion molitor v. superbum Lauffer, 1911a: 34. Barbadillo (Burgos). No Cat. 8759.

Serie tipo: 1 ej.- SINTIPO 을 D. molitor v. superbum Lauff. + Ib. (Hisp.) molitor superbum (Lauf.) E. Vives det. 1977 + D. molitor v. superbum Lauff. Holotipo. Iberodorcadion (Hispanodorcadion) molitor (Fabricius, 1775) [Vives, 2000].

tenuilineatum. D. Graellsi var. tenuilineatum Escalera, 1924: 196. Riofrío. No Cat. 8741.

Serie tipo: 19 ejs.- SINTIPO $ᄋ$ : D. graellsi var. tenuelineatum Esc. Sintipo + Riofrio + Ib. (Hispanod.) graellsi longipenne Esc. Vives det. 1976. 18 SINTIPOS $\left(90^{7} \sigma^{\pi}, 9\right.$ ㅇ $ᄋ$ ) : $D$. graellsi var. tenuelineatum Esc. Sintipo + Riofrio. Iberodorcadion (Hispanodorcadion) graellsii ssp. longipenne (Chevrolat, 1862) [Vives, 2000].

terolense. D. Terolense Escalera, 1902: 282-283. Monteagudo, Escriche, Gúdar, Valdelinares (Teruel). N ${ }^{\circ}$ Cat. 8754.

Serie tipo: 12 ejs.- LECTOTIPO $\sigma^{7}: 23 / 2+$ lectotipo + Gúdar $+D$. terolense Esc. $\sigma^{\pi}$ tipo. PARALECTOTIPO $\sigma^{x}$ : $23 / 3+$ Escriche $+D$. terolense Esc. $\sigma^{\prime}$ tipo + paralectotipo $+I b$. (H.) terolense Vives det. 1977. 8 PARALECTOTIPOS $\left(4 \sigma^{7} \sigma^{7}, 4\right.$ ㅇ $ᄋ$ ) : Escriche $+D$. terolense Esc. $\sigma^{7}$ tipo + paralectotipo [con etiquetas de numeración: 23/4 a 23/6, 23/9 a 23/13; dos de los ejemplares con etiqueta: MNCN MADRID]. 2 PARALECTOTIPOS $\sigma^{\pi} \sigma^{7}$ : Gúdar + paralectotipo $+D$. terolense Esc. ㅇ tipo + Iber. (Hispan.) terolense (Esc.) E. Vives det. 1977 [con etiquetas de numeración: 23/7, 23/8; uno de los ejemplares con etiqueta: MNCN MADRID]. Iberodorcadion (Hispanodorcadion) terolense (Escalera, 1902) [Vives, 1983].

tornavacense. Dorcadion segovianum v. tornavacense Lauffer, 1911b: 52. Puerto de Tornavacas. No Cat. 2347.

Serie tipo: 12 ejs.- 6 SINTIPOS $\left(5 \sigma^{\pi} \sigma^{\pi}, 1\right.$ ㅇ $)$ : Pto. Tornavacas, 6/04. 6 SINTIPOS $\left(40^{7} \sigma^{7}, 2\right.$, 2 \%): Tornavacas a Sierra Gil $G^{\text {a }}$, 5/04. Iberodorcadion (Hispanodorcadion) segovianum (Chevrolat, 1862) [Vives, 2000].

transitorium. Dorcadion Panteli a. transitorium Lauffer, 1911a: 29-30. Uclés. N Cat. 8820.

Serie tipo: 10 ejs.- SINTIPO $\sigma^{\pi}: 43 / 2+$ Uclés + D. uhagoni Per. Uclés [en reverso: Pantel] + col. Sr. Pérez Arcas $+D$. panteli Thery $\sigma^{\prime}$ Esc. Vidit. SINTIPO $\sigma^{*}: D$. panteli a. transitorium Lauff. + Uclés $+D$. panteli ab. transitorium Lauff. Sintipo. SINTIPO $\sigma^{7}$ : Uclés $+D$. panteli ab. transitorium Lauff. Sintipo. 6 SINTIPOS (2 $\sigma^{7} \sigma^{x}, 4$ 우 우): Uclés, Pantel. SINTIPO $Q:$ Pantel [en reverso: Uclés]. Iberodorcadion (Hispanodorcadion) uhagonii (Pérez Arcas, 1868) [Saz, 2007b].

Obs. Nombre no disponible (CINZ Art. 45.5, 45.6.2. Ver aberrantipes).

tristiculum. Dorcadion Dejeani var. montanum a. 우 tristiculum Lauffer, 1911b: 54. Sierra de Béjar. $N^{\circ}$ Cat. 8808.

Serie tipo: 1 ej.- SINTIPO 오 : Sierra de Bejar $+D$. dejeani montanum a. tristiculum ㅇ Lauff. + ab. tristiculum Sintipo.
Iberodorcadion (Hispanodorcadion) segovianum ssp. dejeanii (Chevrolat, 1862) [Vives, 2000].

Obs. Nombre no disponible (CINZ Art. 45.5, 45.6.2. Ver aberrantipes).

turdetanum. Dorcadion turdetanum Lauffer, 1911a: 25-27. Teruel. N ${ }^{\circ}$ Cat. 8740.

Serie tipo: 3 ejs.- LECTOTIPO $\sigma^{\top}:$ D. turdetanum Lauff. + lectotipo + Iber. (Hispanodord.) zarcoi turdetanum Lauf. E. Vives det. 1977 + MNCN MADRID. 2 PARALECTOTIPOS $\left(0^{7}\right.$, O ): paralectotipo + Iber. (Hispanodorc.) zarcoi turdetanum Lauf. E. Vives det. 1977 [uno de los ejemplares con etiqueta: MNCN MADRID]. Iberodorcadion (Hispanodorcadion) zarcoi ssp. turdetanum (Lauffer, 1911) [Vives, 1983].

tutori. Dorcadion molitor v. Tutori Lauffer, 1911a: 34. Calahorra. $\mathrm{N}^{\mathrm{o}}$ Cat. 8758.

Serie tipo: 1 ej.- SINTIPO $\sigma^{n}:$ D. molitor v. tutori Lauff. + Calahorra, Tutor $+I b$. (Hisp.) molitor tutori (Lauff.) E. Vives det. $1977+$ D. molitor v. tutori Lauffer Sintipo. Iberodorcadion (Hispanodorcadion) molitor (Fabricius, 1775) [Vives, 2000].

uhagonii. Dorcadion Uhagonii Pérez Arcas, 1868: 83. Cuenca. $\mathrm{N}^{\circ}$ Cat. 8768.

Serie tipo: 3 ejs.- LECTOTIPO 우 : Tipo de figura + lectótipo $+I b$. (Hispanodorcadion) uhagoni (Pérez Arcas) E. Vives det. 1977 + MNCN Cat. Tipos No 8768 [ejemplar con una etiqueta amarilla]. PARALECTOTIPO o : 41/21 + Cuenca + col. Sr. Pérez Arcas + lectoparatipo + Ib. (Hispanodorcadion) uhagoni (Pérez Arcas) E. Vives det. $1977+$ MNCN Cat. Tipos $\mathrm{N}^{\circ}$ 8768. PARALECTOTIPO 운 $41 / 22$ + Cuenca, D. uhago$n i$ Per. + lectoparatipo + Ib. (Hispanodorcadion) uhagoni (Pérez Arcas) E. Vives det. 1977 + MNCN Cat. Tipos No 8768. Iberodorcadion (Hispanodorcadion) uhagonii (Pérez Arcas, 1868) [Vives, 1983].

umbripenne. D. umbripenne Escalera, 1908: 336-337. Cuenca del Tormes. $\mathrm{N}^{\circ}$ Cat. 2344.

Serie tipo: 1 ej.- SINTIPO $\sigma^{7}: 56 / 1+$ Rio Tormes $+D$. umbripenne $\sigma^{x}$ Esc. Iberodorcadion (Hispanodorcadion) abulense (Lauffer, 1902) [Vives, 1983].

umbrosum. D. umbripenne var. umbrosum Escalera, 1911: 83. [En la descripción original no se indica localidad]. $\mathrm{N}^{\circ}$ Cat. 8771 .

Serie tipo: 14 ejs.- SINTIPO $\sigma^{7}$ : Villatoro, J. Ardois $+I b$. (Hisp.) abulense (Lauff.) E. Vives det. $1977+$ D. umbripenne var. umbrosum Sintipo. SINTIPO $\sigma^{7}$ : Valle del Tormes $+D$. umbripenne var. umbrosum Sintipo. 4 SINTIPOS $\sigma^{\pi} \sigma^{\pi}$ : Villatoro, J. Ardois $+D$. umbripenne var. umbrosum Sintipo. 8 SINTIPOS $\left(60^{\top} \sigma^{\top}, 2\right.$ 우 우): Menga, J. Ardois $+D$. umbripenne var. umbrosum Sintipo [uno de los ejemplares con una etiqueta pequeña blanca circular]. Iberodorcadion (Hispanodorcadion) abulense (Lauffer, 1902) [Vives, 2000].

urbionense. D. almarzense Var. Urbionense: Escalera, 1902: 280. Peñas de Urbión. N ${ }^{\circ}$ Cat. 2343.

Serie tipo: 4 ejs.- SINTIPO $\sigma^{7}$ : Urbión + D. urbionense Esc. + Sintipo + MNCN MADRID. Iberodorcadion (Hispanodorcadion) neilense (Escalera, 1902) [Vives, 2000]. 


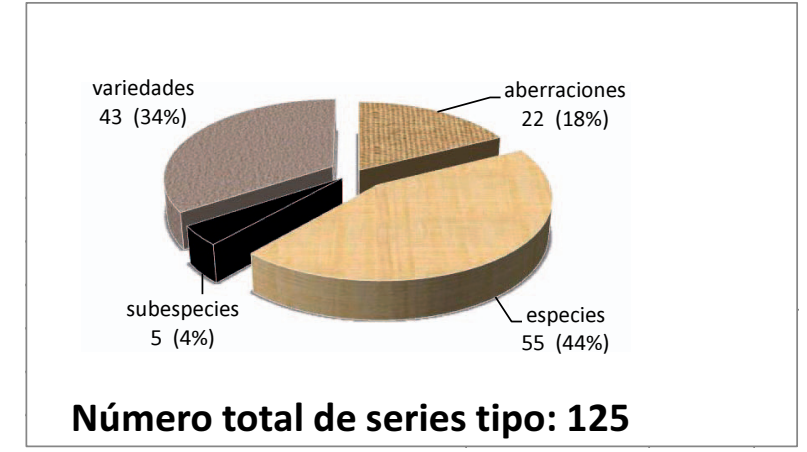

Fig. 1.- Categorías taxonómicas de los táxones representados.

Fig. 1.- Taxonomic categories of the taxa represented in this catalog.

vallisoletanum. Dorcadion vallisoletanum Lauffer, 1901: 89. Valladolid. $\mathrm{N}^{\mathrm{o}}$ Cat. 2341.

Serie tipo: 1 ej.- SINTIPO $\sigma^{x}$ : Valladolid + D. vallisoletanum Lauff. Iberodorcadion (Iberodorcadion) spinolae (Dalman, 1817) [Vives, 1983].

varipes. Dorcadion Martinezi a. varipes Lauffer, 1911a: 32. Madrid. No Cat. 8813.

Serie tipo: 1 ej.- SINTIPO $\sigma^{x}$ : Dorcadion martinezi a. varipes Lauff. + Retiro $+D$. martinezi ab. varipes Lauff. Sintipo. Iberodorcadion (Hispanodorcadion) martinezii (Pérez Arcas, 1874) [Saz, 2007b].

Obs. Nombre no disponible (CINZ Art. 45.5, 45.6.2. Ver aberrantipes).

villosladense. D. Villosladense Escalera, 1902: 280-281. Villoslada, Sierra Cebollera (Logroño). N ${ }^{\circ}$ Cat. 8785.

Serie tipo: 3 ejs.- SINTIPO $\sigma^{x}: \mathrm{S}^{\mathrm{a}}$ Cebollera $+D$. villosladense Esc. $+D$. villosladense $\sigma^{7}$ tipo $+D$. villosladense Esc. Sintipo + MNCN Cat. Tipos No 8785. SINTIPO $0^{x}: 34 / 1+$ Villoslada $+D$. villosladense Esc. Sintipo + MNCN Cat. Tipos $\mathrm{N}^{\circ}$ 8785. SINTIPO 으: Villoslada, 14.5.02 + D. villosladense Esc. Sintipo + MNCN Cat. Tipos $N^{\circ} 8785$. Iberodorcadion (Hispanodorcadion) neilense (Escalera, 1902) [Vives, 2000].

zarcoi. Dorcadion zarcoi Schramm, 1910: 285-286. Epila. No Cat. 8739.

Serie tipo: 12 ejs.- LECTOTIPO $\sigma^{\top}:$ 65/17 + Epila, regalo de A. Zarco + lectotipo + Iber. (Hispanodorc.) zarcoi E. Vives det. $1977+$ Iber. (Hispan.) zarcoi (Schram) E. Vives det. 1977 + MNCN Cat. Tipos No 8739. 6 PARALECTOTIPOS $\sigma^{7} \sigma^{7}$ : Epila, 1910, Zarco + paralectotipo + Iber. (Hispanodorc.) zarcoi (Schram) E. Vives det. $1977+$ MNCN Cat. Tipos $\mathrm{N}^{\mathrm{o}} 8739$ [5 ejemplares con etiquetas de numeración: 65/2,65/3,65/5, 65/7, 65/25]. 2 PARALECTOTIPOS 우 우: Epila, 1910, Zarco + Iber. (Hispanodorc.) zarcoi E. Vives det. 1977 [1 ejemplar con etiqueta de numeración: 65/24]. 3 PARALECTOTIPOS 우 우: Epila, 1910, Zarco [con etiqueta de numeración: 65/12, 65/13, 65/14]. Iberodorcadion (Hispanodorcadion) zarcoi (Schramm, 1910) [Vives, 1983].
Tabla 1.- Autores de los táxones, número de táxones descritos por cada uno e intervalo entre descripciones.

Table 1.- Authors of taxa, number of those described by each of them and interval between first and late descriptions.

\begin{tabular}{lcc}
\hline Autor & N $^{\circ}$ de táxones & años \\
\hline M. Escalera & 57 & $1900-1924$ \\
Lauffer & 47 & $1898-1911$ \\
Graells & 3 & $1849-1858$ \\
Pérez Arcas & 3 & $1868-1874$ \\
Schramm & 3 & $1909-1911$ \\
Verdugo & 2 & $1995-2003$ \\
Aguado \& Tomé & 1 & 1999 \\
Anitchenko \& Verdugo & 1 & 2004 \\
Bahillo & 1 & 1994 \\
Berger & 1 & 1997 \\
Corraleño \& Murría & 1 & 2012 \\
González & 1 & 1992 \\
Pic & 1 & 1899 \\
Saz & 1 & 2007 \\
Tomé \& Bahillo & 1 & 1996 \\
Tomé \& Berger & 1 & 1999 \\
\hline
\end{tabular}

zenete. Iberodorcadion (Hispanodorcadion) zenete Anichtchenko \& Verdugo: 2004: 31-42. Sierra Nevada (Almería). No Cat. 9594.

Serie tipo: 2 ejs.- HOLOTIPO $\sigma^{x}$ : Pto. de la Ragua 2040 m, Bayárcal, Almería, Sierra Nevada, ex larva en Phalaris sp., 26/MAY/2004, Verdugo \& Anichtchenko leg. + Iberodorcadion (H.) zenete, A. \& V. 04, A. Verdugo det. 2004 + HOLOTYPUS Ib. (H.) zenete sp nov. A. Anitchtchenko \& Verdugo det. 2004. PARATIPO ㅇ: Pto. de la Ragua 2040m, Bayárcal, Almería, Sierra Nevada, ex larva en Phalaris sp., 26/MAY/2004, Verdugo \& Anichtchenko leg. + Iberodorcadion (H.) zenete, A. \& V. 04. A. Verdugo det. $2004+$ ALLOTYPUS $I b$. (H.) zenete sp nov. A. Anitchtchenko \& Verdugo det. 2004. Iberodorcadion (Hispanodorcadion) zenete Anichtchenko \& Verdugo, 2004. [Anichtchenko \& Verdugo: 2004].

\section{Conclusiones}

Se han identificado y relacionado 734 ejemplares tipo de Iberodorcadion, correspondientes a 125 táxones (55 especies, 5 subespecies, 43 variedades y 22 aberraciones) descritos por 17 autores, entre los años 1849 y 2012; en la Tabla 1 y las Figuras 1 a 3 se aportan algunos otros datos cuantitativos relativos a este material. Se pueden consultar las fotografías de todos los ejemplares en la web del MNCN (http://www.mncn.csic.es), Colección de Entomología, accediendo al apartado Para saber más: Ejemplares tipo de Iberodorcadion (Breunning). Fotografías. 


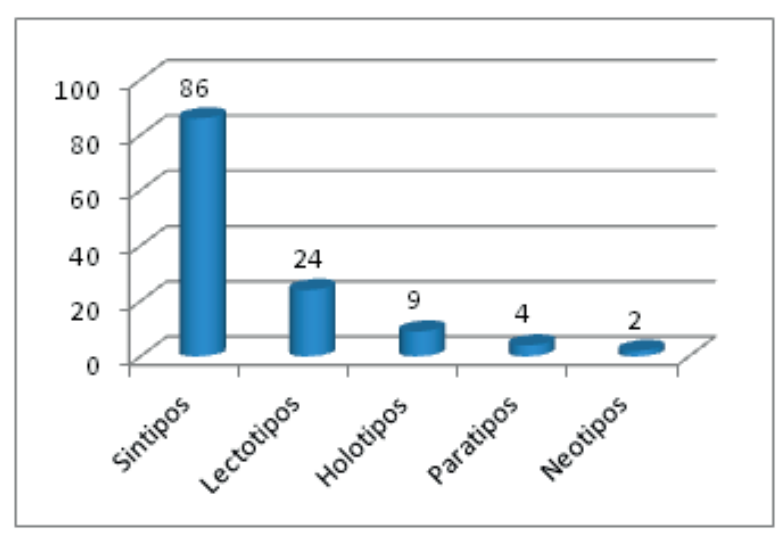

Fig. 2.- Representación de los distintos tipos en las series.

Fig. 2.- Different types represented in the series.

Una parte significativa de estos táxones han sido progresivamente sinonimizados o corresponden a simples variedades, o aberraciones sin valor taxonómico en la actualidad, si bien entendemos que su interés histórico es considerable pues reflejan la extremada variabilidad morfológica presente en este grupo de especies endémicas y el interés que esa misma versatilidad ha venido despertando durante años en buen número de especialistas. El elevado número de estudios existentes a través de los años sobre este género de la familia Cerambycidae de la Península Ibérica y las cuantiosas descripciones de unidades taxonómicas distintas, en intentos de reflejar tal diversidad, justifican la elaboración de catálogos como el que aquí se presenta.

Aparte de ello, es obligado resaltar el valor científico de la colección de Iberodorcadion del Museo Nacional de Ciencias Naturales, que reúne el material tipo de 39 de los 76 táxones, con rango específico o subespecífico, del total peninsular aceptado en el último catálogo de la familia Cerambycidae (González et al., 2007), más tres especies y subespecies rehabilitadas o descritas en publicaciones posteriores, lo que significa el 51,3\% de ese total.

A continuación se relacionan estos táxones, en el mismo orden que se establece en dicha publicación, incluyendo los tres que, como se ha indicado, no figuraban en ella: Iberodorcadion (Hispanodorcadion) ssp. intermedium (Escalera, 1902), descrito como simple variedad y considerado como subespecie por Tomé (2008) y Saz (2010); así como Iberodorcadion (Hispanodorcadion) uhagonii ssp. pradae Saz,

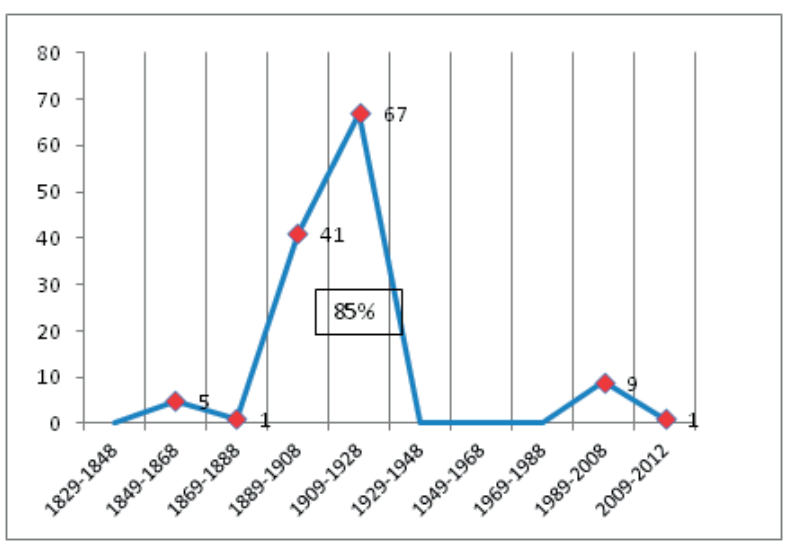

Fig. 3.- Secuencia de la descripción de los táxones. Un alto porcentaje de los mismos, 106, lo fueron en el intervalo 1900-1924, debido principalmente a los estudios de Martínez de la Escalera y Lauffer.

Fig. 3.- Sequence of the taxa descriptions. Most of them, 106, were described between 1900-1924, mainly due to the studies by Martínez de la Escalera and Lauffer.

2007a, e Iberodorcadion (Hispanodorcadion) paulae Corraleño \& Murria, 2012, ambos descritos con posterioridad a la aparición del mencionado Catálogo.

\section{Iberodorcadion (Iberodorcadion)}

fuliginator ssp. loarrense (Berger, 1997)

seoanei (Graells, 1858)

seoanei ssp. laurae Bahillo, 1993

spinolae ssp. caunense (Lauffer, 1910)

\section{Iberodorcadion (Baeticodorcadion)}

amorii ssp. segurense (Escalera, 1911)

coelloi (Verdugo, 1996)

isernii (Pérez Arcas, 1868)

marmottani (Escalera, 1900)

mucidum ssp. rondense Verdugo, 2003

\section{Iberodorcadion (Hispanodorcadion)}

abulense (Lauffer, 1902)

abulense ssp. granulipenne (Escalera, 1908)

aguadoi Aguado \& Tomé, 2000

albicans ssp. demandense (Escalera, 1902)

albicans ssp. marinae (Tomé \& Bahillo, 1996)

albicans ssp. palentinum (Escalera, 1911)

aries Tomé \& Berger, 1999

becerrae (Lauffer, 1901)

bolivari (Lauffer, 1898)

graellsii (Graells, 1858) 
graellsii ssp. cinereum Escalera, 1901

grustani González, 1992

ferdinandi (Escalera, 1900)

fuentei (Pic, 1899)

martinezii (Pérez Arcas, 1874)

molitor ssp. navasi (Escalera, 1900)

mosqueruelense (Escalera, 1902)

neilense (Escalera, 1902)

paulae Corraleño \& Murria, 2012

perezi (Graells, 1849)

perezi ssp. nudipenne (Escalera, 1908)

pseudomolitor (Escalera, 1902)

seguntianum ssp. intermedium (Escalera, 1902)

terolense (Escalera, 1902)

uhagonii (Pérez Arcas, 1868)

uhagonii ssp. pradae Saz, 2007

zarcoi (Schramm, 1910)

zarcoi ssp. turdetanum (Lauffer, 1911)

zenete Anichtchenko \& Verdugo, 2004

\section{Agradecimientos}

El personal de la Colección de Entomología del Museo facilitó enormemente este trabajo y su Responsable, Mercedes París García, aportó también valiosas observaciones sobre algunos ejemplares y series. Miguel Angel Alonso Zarazaga resolvió numerosas dudas de nomenclatura y categorías taxonómicas. Vaya desde aquí nuestro más sincero reconocimiento y estima a todos ellos, así como a los revisores del manuscrito por sus valiosos comentarios.

Trabajo parcialmente financiado por el Proyecto HAR201128621 al segundo autor. graellsii ssp. incallosum (Escalera, 1908)

Breuning, S., 1947. Nouvelles formes de Dorcadion (Coleoptera, Cerambycidae). 2e note. Miscellanea Entomologica, 43(11): 149-172.

Corraleño, A. \& Murria, A., 2012. Descripción de una nueva especie del género Iberodorcadion Breuning, 1943 (Coleoptera, Cerambycidae, Lamiinae) de la Península Ibérica. Biocosme Mésogéen, 29(2): 43-55.

Escalera, M. M., 1900. Descripciones de algunas nuevas especies de Dorcadion. Actas de la Sociedad española de Historia Natural, 29: 232-241.

Escalera, M. M., 1901. Especies españolas del género Dorcadion. Boletín de la Sociedad española de Historia Natural, 1: 81.

Escalera, M. M., 1902. Especies nuevas del género "Dorcadion". Boletín de la Sociedad española de Historia Natural, 2: 278-289.

Escalera, M. M., 1908. Especies nuevas de "Dorcadion" de España. Boletín de la Real Sociedad española de Historia Natural, 8: 334-337.

Escalera, M. M., 1911. Especies nuevas de "Dorcadion" de España. Boletín de la Real Sociedad española de Historia Natural, 11: 80-84.

Escalera, M. M., 1924. Enumeración de las especies españolas de Dorcadion (Col., Ceramb.) del Museo de Madrid, y descripción de algunas formas nuevas. Boletín de la Real Sociedad española de Historia Natural, 24: 191-200.

González, C., 1992. Un nuevo Iberodorcadion (Breuning, 1943) de la provincia de Teruel (Coleoptera Cerambycidae). Zapateri. Revista aragonesa de Entomología, 1(2): 3-8.

González, C. F., Gonzalo, I. \& Velasco, P., 2001. Iberodorcadion (Hispanodorcadion) mosqueruelense var. pseudomolitor (Escalera, 1902), propuesta de nuevo status (Cerambycidae: Dorcadionini). Zapateri. Revista aragonesa de Entomología, 9: 59-64.

\section{Referencias}

Aguado, L. O. \& Tomé, M., 2000. Nuevo Ib Breuning (Coleoptera: Cerambycidae) Ilberodorcadion León: Iberodorcadion aguadoi. Biocosm Breuning [1999], 16(1-2): 67-78.

\section{(Coleoptera:}

Anichtchenko, A. \& Verdugo, A., 2004. Ibe (erambycidae) de (Hispanodorcadion) zenete, nueva espec Castilla y cerambícido (Coleoptera, Cerambycidae) procedente de Sierra Nevada (Andalucía, España). Boletín de la S.E.A., 11: 31-42.

Bahillo, P., 1994. Una nueva subespecie de Iberodorcadion (s. str.) seoanei (Gräells, 1858) (Coleoptera: Cerambycidae). Elytron, [1993], 7: 65-69.

Berger, P., 1997. Une nouvelle espece d'Iberodorcadion Breuning d' Aragon - Espagne (Coleoptera: Cerambycidae): I. loarrense. Biocosme Mésogéen, 14(1): 39-48.
, C. F., Vives, E. \& Zuzarte, A. J. S., 2007. b catálogo de los Cerambycidae (Coleoptera) Península Ibérica, islas Baleares e islas atlánCanarias, Açores y Madeira. Monografías ., vol. 12. Sociedad Entomológica Aragonesa. oza. $211 \mathrm{pp}$

M. de la P., 1849. Description de quelques pagne. Annales de la Société Entomologique de France, $2^{\text {ème }}$ série, 9: 5.

Graells, M. de la P., 1858. Insectos nuevos de España, descubiertos y descritos por el Dr. D. M. P. Graells. Memorias de la Comisión del Mapa Geológico de España: 91-93.

Hernández, J. M., 1996. Variabilidad y biología de los Iberodorcadion Breuning, 1943 de la Sierra de Guadarrama (Coleoptera, Cerambycidae, Lamiinae). Tesis Doctoral. Universidad Complutense de Madrid. $573 \mathrm{pp}$. pptères nouveaux de la faune centrale 
Hernández, J. M., 2000. Estudio multivariante de la genitalia masculina y femenina en seis especies de Iberodorcadion Breuning, 1943 (Coleoptera, Cerambycidae, Lamiinae) de la Comunidad de Madrid (España) y propuesta de nuevas sinonimias para el grupo. Boletín de la Asociación española de Entomología, 24(1-2): 97-129.

Izquierdo, I. \& Martín, C., 2010. Catálogo de los tipos de especies de hexápodos descritas de las islas Canarias, conservados en el Museo Nacional de Ciencias Naturales (Madrid, España). Vieraea, 38: 23-54.

Izquierdo, I., Martín, C., París, M. \& Santos, C., 1997. La colección de Entomología del Museo Nacional de Ciencias Naturales (CSIC). Graellsia, 53: 49-85. doi: 10.3989/graellsia.1997.v53.i0.365.

Lauffer, J., 1898. Dorcadion bolivari sp. nov. Actas de la Socidad española de Historia Natural, 27, apéndice: 107-108.

Lauffer, J., 1901. Notas críticas sobre el género Dorcadion Dalm. Boletín de la Sociedad española de Historia Natural, 1: 89-98.

Lauffer, J., 1902. Vorläufige Diagnose eines neuen spanichen Dorcadion. Societas Entomologica, 17(14): 1.

Lauffer, J., 1910. Nomenklatorische und synonymische Bemerjungen. Boletín de la Real Sociedad española de Historia Natural, 10: 88-92.

Lauffer, J., 1911a. Formas nuevas del género Dorcadion con notas críticas y sinonímicas. Boletín de la Sociedad aragonesa de Ciencias Naturales, 10(1): 25-36.

Lauffer, J., 1911b. Formas nuevas del género Dorcadion con notas críticas y sinonímicas. Boletín de la Sociedad aragonesa de Ciencias Naturales, 10(2): 39-55.

Lauffer, J., 1911c. Formas nuevas del género Dorcadion con notas críticas y sinonímicas. Boletín de la Sociedad aragonesa de Ciencias Naturales, 10(2): 68-74.

Martín, C. \& Izquierdo, I., 2006. Tipos de especies de insectos descritas de la Comunidad de Madrid, conservados en el Museo Nacional de Ciencias Naturales. Inventario preliminar. Graellsia, 62(Número extraordinario): 109-144. doi: 10.3989/graellsia.2006.v62. iExtra. 117

Pérez Arcas, L., 1868. Insectos nuevos o poco conocidos de la fauna española. Anales de la Sociedad española de Historia Natural, 3: 81-83.

Pérez Arcas, L., 1874. Especies nuevas o críticas de la fauna española. Anales de la Sociedad española de Historia Natural, 3: 145-149.

Pic, M., 1899. Description d'un nouveaux Dorcadion d'Espagne. Miscellanea Entomologica, 6(7): 81.

Pic, M., 1900. Synonymies de quelques espèces et variétés de Dorcadion (Col.). Bulletin de la Société entomologique de France, 1900: 404-405.

Rey del Castillo, C. \& Izquierdo Moya, I. 1989. Tipos de especies de Himenópteros descritas por G. Ceballos, en el Museo Nacional de Ciencias Naturales de Madrid. Eos, 65(2): 251-264.

Romero, J., 2002. Iconografía del género Iberodorcadion. Argania editio. Barcelona. 197 pp.

Saz, A. del, 2007a. Descripción de una nueva subespecie de Iberodorcadion de la Península Ibérica (Coleoptera, Cerambycidae). Boletín de la S.E.A., 40: 195-199.

Saz, A. del, 2007b. Los Iberodorcadion Breuning, 1943 de la Península Ibérica (I): Estudio comparativo de algunas especies de la zona central peninsular (Coleoptera, Cerambycidae). Boletín de la S.E.A., 40: 397-408.

Saz, A. del, 2009. Sobre los ejemplares glabros Iberodorcadion Breuning, 1943 con tomento elitral (Coleoptera, Cerambycidae) y estudio de la demo glabra de Iberodorcadion (Hispanodorcadion) pseudomolitor (Escalera, 1902). Boletín de la S.E.A., 45: 127-134.

Saz, A. del, 2010. Los Iberodorcadion Breuning, 1943 de la Península Ibérica (2 ${ }^{\mathrm{a}}$ nota): estudio de Iberodorcadion (Hispanodorcadion) seguntianum (Daniel \& Daniel, 1899) y análisis de la posición sistemática del taxon intermedium Escalera, 1892 (Coleoptera, Cerambycidae). Boletín de la S.E.A., 46: 235-242.

Saz, A. del, 2011a. Revisión de la posición sistemática de los taxones ariasi Chevrolat, 1862 y tenuecinctum Pic, 1898 de Iberodorcadion (Hispanodorcadion) circumcinctum (Chevrolat, 1862) (Coleoptera, Cerambycidae). Boletín de la S.E.A., 48: 321-326.

Saz, A. del, 2011b. Los Iberodorcadion Breuning, 1943 de la Península Ibérica ( $3^{\mathrm{a}}$ nota): estudio de Iberodorcadion (Hispanodorcadion) nudipenne (Escalera, 1908) (Coleoptera, Cerambycidae). Boletín de la S.E.A., 48: 407-415.

Saz, A. del, Simón, A. \& Zapata, J. L., 2004. Localidades de captura de los Iberodorcadion Breuning, 1943 de la colección del Museo Nacional de Ciencias Naturales de Madrid (Coleoptera, Cerambycidae). Consultado en Julio 2012: http://entomología.rediris.es/Iberodorcadion/col/MNCM.html

Schramm, G., 1909. Description d'une nouvelle espèce de «Dorcadion». Boletín de la Real Sociedad española de Historia Natural, 9: 296-298.

Schramm, G., 1910. Nouveaux Dorcadion d'Espagne. Boletín de la Real Sociedad española de Historia Natural, 10: 285-286.

Schramm, G., 1911. Une nouvelle espéce et une nouvelle aberration de Dorcadion d'Espagne. Boletín de la Real Sociedad española de Historia Natural, 11: 306-307.

Tomé, M., 1997. La aventura Dorcadionológica: interpretaciones y exploraciones. Consideraciones en torno a Iberodorcadion (H.) seguntianum Daniel e Iberodorcadion (H.) becerrae Lauffer. Boletín de la S.E.A., 18: 47-52. 
Tomé, M., 1998. Sobre Iberodorcadion (Hispanodorcadion) seguntianum Daniel \& Daniel, Iberodorcadion (Hispanodorcadion) becerrae Lauffer e Iberodorcadion (Hispanodorcadion) ruspolii Breuning. Zapateri. Revista aragonesa de Entomología, 8: 201-212.

Tomé, M., 2004. Rehabilitación de Dorcadion (Iberodorcadion) nudipenne Escalera (Coleoptera, Cerambycidae). Boletín de la S.E.A., 35: 247-249.

Tomé, M., 2008. Nuevos datos sobre Dorcadion (Iberodorcadion) seguntianum (Daniel \& Daniel, 1988) en la Península Ibérica. Boletín de la S.E.A., 43: 399-402.

Tomé, M. \& Bahillo, P., 1996. Descripción de Iberodorcadion marinae $\mathrm{n}$. sp., nuevo Iberodorcadion de España (Coleoptera, Cerambycidae, Lamiinae). Lambillionea, 96(4): 715-721.

Tomé, M. \& Berger, P., 1999. Une nouvelle espèce d'Iberodorcadion Breuning pour la Peninsule Ibérique: I. aries (Coleoptera, Cerambycidae). Lambillionea, 99(3): 393-396.

Tomé, M., 2001a. Iberodorcadion becerrae (Lauffer, 1901). Proyecto Iberodorcadion. Comunidad Virtual de Entomología Rediris. Consultado en Junio 2012: http://entomologia.rediris.es/iberodorcadion/becerrae/ bece MT.htm

Tomé, M., 2001b. Rehabilitación de Dorcadion (Iberodorcadion) almarzense (Escalera, 1902). Proyecto Iberodorcadion. Comunidad Virtual de Entomología Rediris. Consultado en Julio 2012: http://entomologia.rediris.es/iberodorcadion/becerrae/ bece MT.htm

Verdugo, A., 1995. Descripción de un nuevo Iberodorcadion (Breuning, 1943) de la provincia de Cádiz (España); nueva combinación para $I$. ferdinandi (Escalera, 1900) y nuevas claves para el subgénero Baeticodorcadion Vives, 1976 (Coleoptera, Cerambycidae, Lamiinae). Zoologia Baetica, 6: 9-21.

Verdugo, A., 2001a. Iberodorcadion grisescens (Escalera, 1900), nueva sinonimia de Iberodorcadion mus (Rosenhauer, 1856) (Coleoptera, Cerambycidae, Lamiinae). Revista de la Sociedad Gaditana de Historia Natural, 2: 5-16.

Verdugo, A., 2001b. Iberodorcadion mucidum (Dalman, 1817). Proyecto Iberodorcadion. Comunidad Virtual de Entomología. Rediris. Consultado en Junio 2012: http://entomologia.rediris.es/iberodorcadion/mucidum/mucidumAV.htm
Verdugo, A., 2003. Los Iberodorcadion de Andalucía, España. (Coleoptera, Cerambycidae). Revista de la Sociedad Gaditana de Historia Natural, 3: 116-156.

Vives, E., 1983. Revisión del género Iberodorcadion (Coleópteros Cerambícidos). CSIC, Instituto Español de Entomología. Madrid. $171 \mathrm{pp}$.

Vives, E., 2000. Coleoptera: Cerambycidae. En: Fauna Ibérica, vol. 12. Ramos et al. (Eds.). Museo Nacional de Ciencias Naturales, CSIC. Madrid. 716 pp.

Vives, E. y Alonso-Zarazaga, M.A., 2000. Apéndice 1. Nomenclatura: Lista de sinónimos y combinaciones. En: Coleoptera: Cerambycidae. En: Fauna Ibérica, vol. 12. Ramos et al. (Eds.). Museo Nacional de Ciencias Naturales, CSIC. Madrid: 567-661.

Zapata, J. L., 2000. Los fuentei conservados en el Museo Nacional de Ciencias Naturales de Madrid (Coleoptera, Cerambycidae, Dorcadion). Boletín de la S.E.A., 27: 49-50. 